\title{
INHOMOGENEOUS DIOPHANTINE APPROXIMATION OVER FIELDS OF FORMAL POWER SERIES
}

\author{
YANN BUGEAUD, L. SINGHAL, AND ZHENLIANG ZHANG
}

\begin{abstract}
We prove a sharp analogue of Minkowski's inhomogeneous approximation theorem over fields of power series $\mathbb{F}_{q}\left(\left(T^{-1}\right)\right)$. Furthermore, we study the approximation to a given point $y$ in $\mathbb{F}_{q}\left(\left(T^{-1}\right)\right)^{2}$ by the $S L_{2}\left(\mathbb{F}_{q}[T]\right)$-orbit of a given point $\underline{x}$ in $\mathbb{F}_{q}\left(\left(T^{-1}\right)\right)^{2}$.
\end{abstract}

\section{INTRODUCTION}

By using geometry of numbers, Minkowski, improving an earlier result of Tschebychev, established that for any irrational numbers $\theta$ and any real number $\alpha$ not belong to $\mathbb{Z} \theta+\mathbb{Z}$, there exist infinitely many pairs of integer $(p, q)$, with $q \neq 0$, such that

$$
|q \theta-\alpha-p| \leq \frac{1}{4|q|}
$$

see [3] for the details. The value $1 / 4$ is best possible. Recently, Laurent and Nogueira [10] obtained an analogous result for the orbit of $\operatorname{SL}(2, \mathbb{Z})\left(\begin{array}{l}\xi \\ 1\end{array}\right)$ in $\mathbb{R}^{2}$. The purpose of the present paper is to establish analogues in the setting of formal power series of these two results in inhomogeneous approximation.

Let $q$ be a prime power and $\mathbb{F}_{q}$ the finite field of order $q$. Recall that $\mathbb{F}_{q}[T]$ and $K=\mathbb{F}_{q}(T)$ denote the ring of polynomials and the field of rational functions over $\mathbb{F}_{q}$, respectively. Let $K_{\infty}=\mathbb{F}_{q}\left(\left(T^{-1}\right)\right)$ denote the field of formal power series $x=\sum_{i=-n}^{\infty} a_{i} T^{-i}$ over the field $\mathbb{F}_{q}$. We equip $\mathbb{F}_{q}\left(\left(T^{-1}\right)\right)$ with the norm $\|x\|=q^{n}$, where $a_{-n} \neq 0$ is the first non-zero coefficient in the expansion of the non-zero power series $x$. This integer $n$ is called the degree of $x$ and denoted by $\operatorname{deg} x$.

As $K_{\infty}$ is a locally compact group under addition, it comes with a Haar measure $\nu$ defined upto multiplication by a positive constant. We normalize so that $\nu\left(T^{-1} \mathbb{F}_{q}\left[\left[T^{-1}\right]\right]\right)=1$. Abusing notation, we also use $\nu$ to denote the $n$-fold product measure on $K_{\infty}^{n}$ for all $n \geq 1$. The 'integral part' $[x]$ of any element $x$ in $K_{\infty}$ stands for the unique polynomial $P$ for which $x-P$ in $T^{-1} \mathbb{F}_{q}\left[\left[T^{-1}\right]\right]$ and $\langle x\rangle$ refers to $x-[x]$ with its $q$-adic norm denoted as

$$
\|x\|:=|\langle x\rangle| \text {. }
$$

The norm $|\underline{\theta}|$ of any $\underline{\theta}={ }^{t}\left(\theta_{1}, \ldots, \theta_{n}\right) \in K_{\infty}^{n}$ equals $\max _{i} q^{\operatorname{deg} \theta_{i}}$ and its supremum distance from the nearest $\underline{P}$ in $\mathbb{F}_{q}[T]^{n}$ is denoted by $\|\underline{\theta}\|_{K_{\infty}}$. The subscript $K_{\infty}$ in the norm expressions will be hidden from now on and we hope it will be clear from the context as to which norm is being referred to.

Over the fields of formal power series, there are many results concerning the metrical properties for the inhomogeneous diophantine approximation sets, such as [4, 8, 9, 11], but it seems that the analogue of Minkowski's inhomogeneous

Key words and phrases. Asymptotic and uniform exponents, Diophantine approximation. 
approximation theorem has not yet been published. The following theorem can also be seen as the inhomogeneous version of Dirichlet's Theorem, which says that for every power series $\xi$ in $K_{\infty} \backslash K$, there exist infinitely many polynomials $Q, P \in \mathbb{F}_{q}[T]$ such that

$$
\|Q \xi\| \leq \frac{1}{q|Q|}
$$

see Lemma 2.2 below.

Theorem 1.1. If $\xi \in K_{\infty} \backslash K$ and $\alpha \notin \mathbb{F}_{q}[T]+\mathbb{F}_{q}[T] \xi$, then there exist infinitely many $Q$ in $\mathbb{F}_{q}[T]$ such that

$$
\|Q \xi-\alpha\| \leq \frac{1}{q^{2}|Q|} .
$$

In addition, the factor $q^{2}$ is best possible. Namely, there exists $\xi$ in $T^{-1} \mathbb{F}_{q}\left[\left[T^{-1}\right]\right] \backslash$ $K$ for which

$$
\left\|Q \xi-T^{-1}(1-\xi)\right\| \geq \frac{1}{q^{2}|Q|}
$$

whenever $Q \in \mathbb{F}_{q}[T] \backslash\{0\}$.

The second part of the above theorem is the power series analogue of Theorem IIA of [3, pp. 48-51]. A simple corollary is that the set $\left\{\langle Q \xi\rangle \mid Q \in \mathbb{F}_{q}[T]\right\}$ is dense in $T^{-1} \mathbb{F}_{q}\left[\left[T^{-1}\right]\right]$. This is also true for approximating any vector $\xi \in K_{\infty}^{m}$ by elements belonging to the subgroup $A \mathbb{F}_{q}[T]^{n}+\mathbb{F}_{q}[T]^{m}$ for generic $m \times \bar{n}$ matrices $A$ and follows from [2, Theorem 1.1]. On a slightly different note, we can show that density in the one-dimensional unit ball is achieved by only taking fractional parts of monic polynomial multiples of $\xi$ as opposed to all polynomial multiples.

Proposition 1.2. Let $\xi \in K_{\infty} \backslash K$ and $\alpha$ be arbitrary. Then, there exist infinitely many monic polynomials $Q$ such that

$$
|Q \xi-\alpha-P| \leq \frac{1}{q|Q|}
$$

for some $P$ in $\mathbb{F}_{q}[T]$.

Following [1, 2], we introduce several exponents of homogeneous and inhomogeneous Diophantine approximation. Let $n$ and $m$ be positive integers and $A$ a matrix in $\mathcal{M}_{n, m}\left(K_{\infty}\right)$. Let $\underline{\theta}$ be in $K_{\infty}^{n}$. We denote by $\omega(A, \underline{\theta})$ the supremum of the real numbers $\omega$ for which, for arbitrarily large real numbers $H$, the inequalities

$$
\|A \underline{x}-\underline{\theta}\| \leq H^{-\omega} \text { and }|\underline{x}| \leq H
$$

have a solution $\underline{x}$ in $\mathbb{F}_{q}[T]^{m}$. Let $\widehat{\omega}(A, \underline{\theta})$ be the supremum of the real numbers $\omega$ for which, for all sufficiently large positive real numbers $H$, the inequalities (1.3) have a solution $\underline{x}$ in $\mathbb{F}_{q}[T]^{m}$. The homogeneous exponents $\omega(A)$ and $\widehat{\omega}(A)$ are similarly defined by taking $\underline{\theta}=\underline{0}$ and disallowing $\underline{Q}$ to be so. It is then clear that for any pair $A, \underline{\theta}$, we have $\omega(A, \underline{\theta}) \geq \widehat{\omega}(A, \underline{\theta}) \geq 0$ and $\omega(A) \geq \widehat{\omega}(A) \geq 0$.

In language of exponent defined above, we conclude from Theorem 1.1 that $\omega(\xi, \alpha) \geq 1$ for any irrational $\xi$, and that $\omega(\xi, \alpha)=1$ for some irrational $\xi$ and $\alpha$. We further have

$$
\omega(\underline{\theta})=\widehat{\omega}(\underline{\theta})=1 / m \text { for almost every } \underline{\theta} \in K_{\infty}^{m},
$$

(with respect to the Haar measure) by the Borel-Cantelli lemma. 
There is a lot of recent activity about understanding the Diophantine properties of group actions on homogeneous spaces. If we consider Theorem 1.1 as the study of the action of $\mathbb{F}_{q}[T]$ on $K_{\infty}$, Our next goal is to obtain an analogous result for the standard action of $\mathrm{SL}_{2}\left(\mathbb{F}_{q}[T]\right)$ on $K_{\infty}^{2}$.

Ghosh. Gorodnik, and Nevo [5, 6] have studied the generic rate of approximation by lattice orbits for a large class of lattice actions on homogeneous varieties of connected almost simple, semisimple algebraic groups. Laurent and Nogueira [10] confined their investigations to the standard linear action of the lattice $\operatorname{SL}(2, \mathbb{Z})$ on the punctured plane $\mathbb{R}^{2} \backslash\{\underline{0}\}$. In a previous work [16], the second-named author extended their approach and showed similar results for a few lattices inside $\mathrm{SL}(2, \mathbb{C})$ acting linearly on $\mathbb{C}^{2} \backslash\{\underline{0}\}$. The last two approaches involve making use of some continued fraction algorithm to construct certain convergent matrices belonging to the relevant lattice. An alternate strategy deployed in [12] and [13] works for many more examples as it uses effective equidistribution results but usually gives weaker estimates.

Let $\underline{x}=\left(x_{1}, x_{2}\right) \in K_{\infty}^{2}$ with "slope" $\xi:=x_{1} / x_{2}$ in $K_{\infty}$. We consider its orbit under the standard action of $\mathrm{SL}_{2}\left(\mathbb{F}_{q}[T]\right)$. If the slope $\xi$ is in $K$ and $P / Q$ is its representation in 'lowest terms' for some $Q \in \mathbb{F}_{q}[T] \backslash\{0\}$, the coordinates of any non-zero vector $\gamma \underline{x}$ shall have entries with absolute value at least $\min \left\{\left|x_{2}\right|, 1\right\}$. $|Q|^{-1}$. Similarly, two distinct points in the $\mathrm{SL}_{2}\left(\mathbb{F}_{q}[T]\right)$-orbit of $\underline{x}$ will also be at least $\min \left\{\left|x_{2}\right|, 1\right\} \cdot|Q|^{-1}$ apart and we will have a discrete orbit at hand. We are more interested in analyzing the nature of dense orbits here. Therefore, we assume henceforth that $\xi \in K_{\infty} \backslash K$. Our target is to reach as close to some fixed point $y \in K_{\infty}^{2}$ as possible with the help of smallest matrix size $|\gamma|$ for some $\gamma \in \mathrm{SL}_{2}\left(\mathbb{F}_{q}[T]\right)$. Just like Definition of above exponent, we have

Definition 1.3. The asymptotic Diophantine exponent $\mu(\underline{x}, \underline{y})$ refers to

$$
\sup \left\{\mu|| \gamma \underline{x}-\left.\underline{y}|\leq| \gamma\right|^{-\mu} \text { has inf. many solutions in } \gamma \in \mathrm{SL}_{2}\left(\mathbb{F}_{q}[T]\right)\right\} \text {, }
$$

and the uniform Diophantine exponent $\widehat{\mu}(\underline{x}, \underline{y})$ is given by

$$
\sup \left\{\hat{\mu} \mid \forall H \gg 0, \exists \gamma \in \mathrm{SL}_{2}\left(\mathbb{F}_{q}[T]\right) \text { such that }|\gamma| \leq H,|\gamma \underline{x}-\underline{y}| \leq H^{-\hat{\mu}}\right\} .
$$

Here we give the analogue of the results in Laurent and Nogueira 10].

Theorem 1.4. Let $\underline{x}={ }^{t}\left(x_{1}, x_{2}\right) \in K_{\infty}^{2}$ such that $\xi=x_{1} / x_{2}$ is not in $K$.

(1) We have

$$
\mu(\underline{x}, \underline{0})=1 \quad \text { and } \quad \widehat{\mu}(\underline{x}, \underline{0})=1 / \omega(\xi) .
$$

(2) If the vector $\underline{y}={ }^{t}\left(y_{1}, y_{2}\right) \in K_{\infty}^{2}$ has slope $y=y_{1} / y_{2}$ in $K$, then we have

$$
\mu(\underline{x}, \underline{y})=\frac{\omega(\xi)}{\omega(\xi)+1} \quad \text { and } \quad \widehat{\mu}(\underline{x}, \underline{y})=\frac{1}{\omega(\xi)+1} .
$$

(3) If the slope $y$ of the vector $\underline{y}$ is not in $K$, then we have

$$
\mu(\underline{x}, \underline{y}) \geq \frac{1}{3} \quad \text { and } \quad \widehat{\mu}(\underline{x}, \underline{y}) \geq \frac{\omega(y)+1}{2(2 \omega(y)+1) \omega(\xi)} \geq \frac{1}{4 \omega(\xi)} .
$$

A generic upper bound for the asymptotic exponent $\mu(\underline{x}, \underline{y})$ is given by the following theorem, which is the analogue of Theorem 3 in Laurent and Nogueira [10]. 
Theorem 1.5. Let $\underline{x}$ be a point in $K_{\infty}^{2}$ with irrational slope and let $y$ be an irrational element in $K_{\infty}$ having irrationality exponent $\omega(y)=1$. Then we have

$$
\mu(\underline{x}, \underline{y}) \leq \frac{1}{2}
$$

for almost all points $\underline{y}$ of the line $K_{\infty}^{t}(\underline{y}, 1)$.

In the next section, we present some auxiliary results of continued fraction. In section 3, the analogue of the Minkowski's theorem is proved. The last section is devoted to giving the proof of Theorem 1.4 and Theorem 1.5 .

\section{Continued FRACTIONS}

Let $\left\{A_{i}\right\}_{i \in \mathbb{N}} \subset \mathbb{F}_{q}[T]$ with $\operatorname{deg} A_{i}>0$ for $i>0$. The continued fraction $\xi:=$ $\left[A_{0} ; A_{1}, \cdots\right]$ is the limit of the sequence of partial fractions

$$
\frac{P_{n}}{Q_{n}}:=A_{0}+\frac{1}{A_{1}+\frac{1}{A_{2}+\frac{1}{\ddots+\frac{1}{A_{n}}}}}
$$

as $n \rightarrow \infty$. It exists for every such sequence of partial quotients $A_{i}$ 's and moreover

$$
\left|Q_{n} \xi-P_{n}\right|=\frac{1}{\left|Q_{n+1}\right|} \text {. }
$$

Conversely, given $\xi \in K_{\infty}$, such an expansion is unique. The rational functions $P_{n} / Q_{n}$ are called $n$-th order convergents to $\xi$. They satisfy the recurrence

$$
\begin{array}{lll}
P_{-2}:=0, & P_{-1}:=1, & P_{n}=A_{n} P_{n-1}+P_{n-2} \text { for } n \geq 0, \text { and } \\
Q_{-2}=1, & Q_{-1}=0, & Q_{n}=A_{n} Q_{n-1}+Q_{n-2} \text { for } n \geq 0 .
\end{array}
$$

Every finite expression $\left[A_{0} ; A_{1}, \cdots, A_{N}\right]$ leads to an element of $K$. In the converse direction, it is also true that the continued fraction expansion of every rational function $P / Q$ terminates in finitely many steps because the Euclidean nature of the $\operatorname{ring} \mathbb{F}_{q}[T]$. By induction, $Q_{n} P_{n-1}-P_{n} Q_{n-1}=(-1)^{n}$ and $\left|P_{n}\right|>\left|P_{n-1}\right|$ whenever all the terms are defined. As a consequence, $\left|P_{n}\right|=\left|A_{n} P_{n-1}\right|=\prod_{i=0}^{n}\left|A_{i}\right|$. One can use a similar argument to establish

$$
\left|Q_{n}\right|=\left|A_{n} Q_{n-1}\right|=\prod_{i=1}^{n}\left|A_{i}\right| .
$$

For future use, it follows from (2.2) and (2.4) that

$$
\left|Q_{n}\left(Q_{n} \xi-P_{n}\right)\right|=1 /\left|A_{n+1}\right| \text {. }
$$

Notation 2.1. When $A_{0}=0$ as will often be the case, we simply drop its mention along with the succeeding semi-colon and write $\left[A_{1}, A_{2}, \cdots\right]:=\left[0 ; A_{1}, A_{2}, \cdots\right]$ while $\left[A_{1}, A_{2}, \cdots, A_{n}\right]$ shall denote the corresponding finite truncations.

It is well known that for every $\xi \in K_{\infty}$, there exist infinitely many polynomials $Q$ such that $|Q \xi-P|<1 /|Q|$ for some $P \in \mathbb{F}_{q}[T]$ depending upon $Q$. The next statement from [15] tells us where to look for them.

Lemma 2.2 (Schmidt). For any $Q \in \mathbb{F}_{q}[T] \backslash\{0\}$ such that $|Q \xi-P|<1 /|Q|$, the rational function $P / Q$ is a convergent to $\xi$. 
In particular, these convergents are the best approximants of second kind [7]. What this means is that for all polynomials $Q$ with $0<|Q|<\left|Q_{n+1}\right|$, we have $|Q \xi-P| \geq\left|Q_{n} \xi-P_{n}\right|$ for all $P \in \mathbb{F}_{q}[T]$. For if not, let $P, Q\left(0<|Q|<\left|Q_{n+1}\right|\right)$ satisfy

$$
|Q \xi-P|<\left|Q_{n} \xi-P_{n}\right|=\frac{1}{\left|Q_{n+1}\right|}<\frac{1}{|Q|} .
$$

Then, Schmidt says that such a $P / Q=P_{m} / Q_{m}$ for some $m \leq n$ which would imply

$$
|Q \xi-P| \geq\left|Q_{m} \xi-P_{m}\right|=\frac{1}{\left|Q_{m+1}\right|} \geq \frac{1}{\left|Q_{n+1}\right|}
$$

as $m \leq n$ and recalling (2.4). This however contradicts the assumption in (2.5). Note that if all the $A_{i}$ 's in the continued fraction expansion of $\xi$ were to be linear polynomials over $\mathbb{F}_{q}$, we see that the constant on the right side in (1.2) cannot be improved for uncountably many $\xi$ 's corresponding to the sequences $\left(A_{i}\right) \in\{T, T+1\}^{\mathbb{N}}$.

The quantity $\omega(\xi)$ is also known as the irrationality measure of $\xi \in K_{\infty} \backslash K$. If $\omega \leq 1$, then the denominator sequences $\left\{Q_{n}\right\}$ are strictly increasing in size and $\left|Q_{n+1}\right| \geq\left|Q_{n}\right|^{\omega}$ for all $n \in \mathbb{N}$ trivially. When $1<\omega<\omega(\xi)$, we have $|Q \xi-P| \leq|Q|^{-\omega}$ for infinitely many non-zero polynomials $Q$ and $P \in \mathbb{F}_{q}[T]$. For all non-constant polynomials, $|Q|^{-\omega}<|Q|^{-1}$. Lemma 2.2 then says that any such fraction $P / Q$ has to be a convergent to $\xi$. In other words, both $P$ and $Q$ are a non-zero polynomial multiple of some pair $\left(P_{n}, Q_{n}\right)$ and we conclude that for $1<\omega<\omega(\xi)$ and $Q \in \mathbb{F}_{q}[T]$ with $|Q| \gg 1$,

$$
\frac{1}{\left|Q_{n+1}\right|} \leq\left|R\left(Q_{n} \xi-P_{n}\right)\right|=|Q \xi-P| \leq \frac{1}{|Q|^{\omega}} \leq \frac{1}{\left|Q_{n}\right|^{\omega}}
$$

where $R \in \mathbb{F}_{q}[T] \backslash\{0\}$. The bottomline is $\left|Q_{n+1}\right| \geq\left|Q_{n}\right|^{\omega}$ for infinitely many $n$ 's. Lastly if $\omega>\omega(\xi)$, then in particular, $\left|Q_{n} \xi-P_{n}\right|>\left|Q_{n}\right|^{-\omega}$ for all $n$ large enough. On the other hand, we know its exact value to be $\left|Q_{n+1}\right|^{-1}$. We get that $\left|Q_{n+1}\right|<\left|Q_{n}\right|^{\omega}$ for all $n \gg 1$.

\section{INHOMOGENEOUS APPROXIMATION}

This section is largely dedicated towards obtaining an analogous version of Minkowski's theorem in the field of formal power series. Before that, we follow the proof route in the real case to establish some results related to geometry of numbers.

Lemma 3.1. Let $\theta, \varphi, \psi(\neq 0)$ and $\chi$ be four formal Laurent series over $\mathbb{F}_{q}$ with

$$
\max \{|\theta \chi-\varphi \psi|,|\psi \chi|\} \leq \Delta
$$

for some $\Delta>0$. Then, there exists $P \in \mathbb{F}_{q}[T]$ satisfying

$$
|\theta+P \psi||\varphi+P \chi| \leq q^{-1} \Delta \text { as well as }|\theta+P \psi| \leq|\psi| \text {. }
$$

Proof. Clearly, $\operatorname{deg}\left(\theta+P_{0} \psi\right)<\operatorname{deg} \psi$ for some $P_{0} \in \mathbb{F}_{q}[T]$. We let $\theta^{\prime}:=\theta+P_{0} \psi$ and $\varphi^{\prime}:=\varphi+P_{0} \chi$, respectively so that $\left|\theta^{\prime} \chi-\varphi^{\prime} \psi\right| \leq \Delta$.

Case 1. If $\left|\varphi^{\prime}\right| \leq|\chi|$, we have imitating [3]

$$
16\left|\theta^{\prime} \varphi^{\prime}\right|\left|\left(\theta^{\prime}+\psi\right)\left(\varphi^{\prime}+\chi\right)\right| \leq\left(\left|\theta^{\prime}\right|+\left|\theta^{\prime}+\psi\right|\right)^{2}\left(\left|\varphi^{\prime}\right|+\left|\varphi^{\prime}+\chi\right|\right)^{2} .
$$


By our construction, $\left|\theta^{\prime}+\psi\right| \leq|\psi|$ and so is $\left|\theta^{\prime}\right|$. We assumed $\left|\varphi^{\prime}\right| \leq|\chi|$ which also implies that $\left|\varphi^{\prime}+\chi\right| \leq|\chi|$. The conclusion is that

$$
\left|\theta^{\prime} \varphi^{\prime}\right|\left|\left(\theta^{\prime}+\psi\right)\left(\varphi^{\prime}+\chi\right)\right|<|\psi|^{2}|\chi|^{2} \leq \Delta^{2} .
$$

Case 2. Else if $\left|\varphi^{\prime}\right|>|\chi|$, it follows that $\left|\theta^{\prime} \chi\right|<\left|\varphi^{\prime} \psi\right|$ which in turn means that $\left|\theta^{\prime} \chi-\varphi^{\prime} \psi\right|=\left|\varphi^{\prime} \psi\right|$. The A. M. - G. M. inequality dictates

$$
\begin{aligned}
2\left(\left|\theta^{\prime} \varphi^{\prime}\right|\left|\left(\theta^{\prime}+\psi\right)\left(\varphi^{\prime}+\chi\right)\right|\right)^{\frac{1}{2}} & \leq\left|\varphi^{\prime}\left(\theta^{\prime}+\psi\right)\right|+\left|\theta^{\prime}\left(\varphi^{\prime}+\chi\right)\right| \\
& \leq\left|\varphi^{\prime} \psi\right|+\left|\theta^{\prime} \varphi^{\prime}\right|<2 \Delta .
\end{aligned}
$$

The equations (3.4) and (3.5) together give us that $\min \left\{\left|\theta^{\prime} \varphi^{\prime}\right|,\left|\left(\theta^{\prime}+\psi\right)\left(\varphi^{\prime}+\chi\right)\right|\right\}$ is less than $\Delta$. We remind the reader that $\max \left\{\left|\theta^{\prime}\right|,\left|\theta^{\prime}+\psi\right|\right\} \leq|\psi|$. Otherwise said, one of the substitutions $P=P_{0}$ or $P=P_{0}+1$ in (3.2) proves our claim.

In the proof, we need the following version of Minkowski's linear forms theorem.

Theorem $3.2([17,18])$. Let $A=\left(a_{i, j}\right)_{n \times n}$ be an $n \times n$ matrix with entries in $K_{\infty}$ and $\underline{r}=\left(r_{1}, r_{2}, \cdots, r_{n}\right)$ be an $n$-tuple of integers. If

$$
0<|\operatorname{det}(A)|<q^{-\left(r_{1}+r_{2}+\cdots+r_{n}\right)},
$$

then there is a non-zero integral point $\underline{u}$ such that $\left|L_{i}(\underline{u})\right|<q^{-r_{i}}$ for all $1 \leq i \leq n$, where the linear forms $L_{i}(\underline{x})$ are determined by the rows of the matrix $A$.

Theorem 3.3. Let $L_{j}(\underline{P}):=\lambda_{j} P_{1}+\kappa_{j} P_{2}$ for $j=1,2$ and $\Delta=\left|\lambda_{1} \kappa_{2}-\lambda_{2} \kappa_{1}\right|>0$.

(1) For all $\rho_{1}, \rho_{2} \in K_{\infty}, \exists \underline{Q} \in \mathbb{F}_{q}[T]^{2}$ such that

$$
\left|L_{1}(\underline{Q})+\rho_{1}\right|\left|L_{2}(\underline{Q})+\rho_{2}\right| \leq q^{-2} \Delta .
$$

(2) If moreover $\kappa_{1} \lambda_{1}^{-1} \notin K$ and $k \in \mathbb{N}$, there exists $\underline{Q}$ which also makes

$$
\operatorname{deg}\left(L_{1}(\underline{Q})+\rho_{1}\right)<-k \text {. }
$$

Proof. Assume $\kappa_{1} \lambda_{1}^{-1} \notin K$ to begin with. Theorem 3.2 tells us that there is a non-zero $\underline{P}=\left(P_{1}, P_{2}\right) \in \mathbb{F}_{q}[T]^{2}$ for which

$$
\left|L_{1}(\underline{P})\right|<q^{-k} \text {, and }\left|L_{2}(\underline{P})\right| \leq q^{k} \Delta .
$$

We can assume that $\operatorname{gcd}\left(P_{1}, P_{2}\right)=1$ without any loss of generality. Therefore, pick any $\left(R_{1}, R_{2}\right) \in \mathbb{F}_{q}[T]^{2}$ for which

$$
\operatorname{det}\left(\begin{array}{ll}
P_{1} & R_{1} \\
P_{2} & R_{2}
\end{array}\right)=1
$$

and we have the transformed system

$$
\left(\begin{array}{l}
L_{1}(\underline{Q}) \\
L_{2}(\underline{Q})
\end{array}\right)=\left(\begin{array}{ll}
\lambda_{1} & \kappa_{1} \\
\lambda_{2} & \kappa_{2}
\end{array}\right)\left(\begin{array}{l}
Q_{1} \\
Q_{2}
\end{array}\right)=\left(\begin{array}{ll}
\lambda_{1}^{\prime} & \kappa_{1}^{\prime} \\
\lambda_{2}^{\prime} & \kappa_{2}^{\prime}
\end{array}\right)\left(\begin{array}{l}
Q_{1}^{\prime} \\
Q_{2}^{\prime}
\end{array}\right)
$$

for all $\underline{Q}=\left(Q_{1}, Q_{2}\right) \in \mathbb{F}_{q}[T]^{2}$, where

$$
\left(\begin{array}{l}
Q_{1}^{\prime} \\
Q_{2}^{\prime}
\end{array}\right):=\left(\begin{array}{ll}
P_{1} & R_{1} \\
P_{2} & R_{2}
\end{array}\right)^{-1}\left(\begin{array}{l}
Q_{1} \\
Q_{2}
\end{array}\right) .
$$

It is plain that $\left(Q_{1}^{\prime}, Q_{2}^{\prime}\right) \in \mathbb{F}_{q}[T]^{2}$ if and only if $\left(Q_{1}, Q_{2}\right)$ does too and also that $\operatorname{det}\left(\begin{array}{ll}\lambda_{1} & \kappa_{1} \\ \lambda_{2} & \kappa_{2}\end{array}\right)=\operatorname{det}\left(\begin{array}{cc}\lambda_{1}^{\prime} & \kappa_{1}^{\prime} \\ \lambda_{2}^{\prime} & \kappa_{2}^{\prime}\end{array}\right)$ owing to (3.9) . Further, $\left|\lambda_{1}^{\prime}\right|=\left|L_{1}(\underline{P})\right|<q^{-k}$ and similarly $\left|\lambda_{2}^{\prime}\right| \leq q^{k} \Delta$ from (3.8), (3.10) and (3.11). The former is non-zero since 

$\kappa_{1} \lambda_{1}^{-1}$ was taken to be an irrational function in $T^{-1}$.

Let $Q_{2}^{\prime} \in \mathbb{F}_{q}[T]$ be such that $\left|\rho_{1} \lambda_{2}^{\prime}-\rho_{2} \lambda_{1}^{\prime}-Q_{2}^{\prime}\left(\lambda_{1} \kappa_{2}-\lambda_{2} \kappa_{1}\right)\right| \leq q^{-1} \Delta$. Also, we substitute in Lemma 3.1

$$
\begin{array}{r}
\theta=\kappa_{1}^{\prime} Q_{2}^{\prime}+\rho_{1}, \quad \varphi=\kappa_{2}^{\prime} Q_{2}^{\prime}+\rho_{2} \\
\psi=\lambda_{1}^{\prime}, \quad \text { and } \quad \chi=\lambda_{2}^{\prime}
\end{array}
$$

to get that $|\theta \chi-\psi \varphi|=\left|\rho_{1} \lambda_{2}^{\prime}-\rho_{2} \lambda_{1}^{\prime}-Q_{2}^{\prime}\left(\lambda_{1} \kappa_{2}-\lambda_{2} \kappa_{1}\right)\right| \leq q^{-1} \Delta$ as well as $|\psi \chi|=\left|\lambda_{1}^{\prime} \lambda_{2}^{\prime}\right| \leq q^{-1} \Delta$. We already argued $\psi \neq 0$. Thus, there exists some $Q_{1}^{\prime} \in \mathbb{F}_{q}[T]$ (and the associated pair $\left(Q_{1}, Q_{2}\right)$ determined by (3.11) $)$ for which

$$
\begin{aligned}
\left|L_{1}(\underline{Q})+\rho_{1}\right|\left|L_{2}(\underline{Q})+\rho_{2}\right| & =\left|\lambda_{1}^{\prime} Q_{1}^{\prime}+\kappa_{1}^{\prime} Q_{2}^{\prime}+\rho_{1}\right|\left|\lambda_{2}^{\prime} Q_{1}^{\prime}+\kappa_{2}^{\prime} Q_{2}^{\prime}+\rho_{2}\right| \\
& =\left|\theta+\psi Q_{1}^{\prime}\right|\left|\varphi+\chi Q_{1}^{\prime}\right| \leq q^{-2} \Delta \text { and } \\
\left|L_{1}(\underline{Q})+\rho_{1}\right| & =\left|\theta+\psi Q_{1}^{\prime}\right| \leq|\psi|<q^{-k} .
\end{aligned}
$$

If it happens that $\lambda_{1} P_{1}+\kappa_{1} P_{2}=0$ (when $\kappa_{1} \lambda_{1}^{-1} \in K$ ), we can be sure that $\lambda_{2} P_{1}+\kappa_{2} P_{2} \neq 0$ as $\Delta \neq 0$. Hence, we only need to exchange the roles of $L_{1}$ and $L_{2}$ amongst themselves and the conclusion in (3.6) remains valid.

The constant on the right side in (3.6) is the smallest possible in general as follows easily from the observation that $\left|P_{1}+T^{-1}\right|\left|P_{2}+T^{-1}\right| \geq q^{-2}$ for all $P_{1}, P_{2} \in$ $\mathbb{F}_{q}[T]$ (here $\Delta=1$ ). We are now in a position to prove the promised version of Minkowski's result on inhomogeneous Diophantine approximation.

Proof of Theorem 1.1. Let $L_{1}(\underline{Q})=\xi Q_{1}+Q_{2}, L_{2}(\underline{Q})=Q_{1}, \rho_{1}=\alpha$ and $\rho_{2}=0$ in Theorem 3.3. We have $\Delta=1$ and as $\xi \notin K$ and $\alpha \neq Q \xi+P$ for any $P, Q \in \mathbb{F}_{q}[T]$, the solution set $\left\{\left(Q_{1}^{(k)}, Q_{2}^{(k)}\right)\right\}$ to (3.6) corresponding to different $k$ in (3.7) is infinite.

Now we turn to prove the second part. Let $\xi=\left[A_{1}, A_{2}, \cdots\right]$ such that $\operatorname{deg} A_{i}>0$ for all $i \geq 1$ and $T \mid A_{i}$ in $\mathbb{F}_{q}[T]$ for all $i$. It is clear that $|\xi|=1 /\left|A_{1}\right|$. Now, suppose

$$
q^{2}|Q(Q \xi-\alpha-P)|=|T Q||(T Q+1) \xi-(T P+1)|<1
$$

for some non-zero polynomial $Q$ and $P \in \mathbb{F}_{q}[T]$. Then, $|T Q+1|$ has to be at least $\left|A_{1}\right|=\left|Q_{1}\right| \geq\left|Q_{1} Q_{0}\right|^{1 / 2}$ or else, $|(T Q+1) \xi|<1$ rendering (3.14) untrue. We thereby have a unique $n \geq 1$ such that

$$
\left|Q_{n} Q_{n-1}\right|^{1 / 2} \leq|T Q|=|T Q+1|<\left|Q_{n+1} Q_{n}\right|^{1 / 2} .
$$

As $\left|P_{n-1} Q_{n}-P_{n} Q_{n-1}\right|=1$, we have a unique pair $(U, V) \in \mathbb{F}_{q}[T]^{2}$ satisfying

$$
\left(\begin{array}{ll}
P_{n-1} & P_{n} \\
Q_{n-1} & Q_{n}
\end{array}\right)\left(\begin{array}{l}
U \\
V
\end{array}\right)=\left(\begin{array}{l}
T P+1 \\
T Q+1
\end{array}\right)
$$

which obey

$$
\begin{aligned}
|U| & =\left|(T P+1) Q_{n}-P_{n}(T Q+1)\right| \\
& =\left|(T Q+1)\left(Q_{n} \xi-P_{n}\right)-Q_{n}((T Q+1) \xi-(T P+1))\right|<\left|A_{n}\right|^{1 / 2}
\end{aligned}
$$

and using (3.15) again,

$$
|V|=\left|(T P+1) Q_{n-1}-P_{n-1}(T Q+1)\right|<\left|A_{n+1}\right|^{1 / 2} .
$$


Our assumption about the partial quotients $A_{i}$ 's of $\xi$ gives that exactly one of the polynomials $P_{n}$ and $Q_{n}$ is divisible by $T$ in $\mathbb{F}_{q}[T]$ for each $n$. This implies neither $U$ nor $V$ can be zero in (3.16) and in turn that $|U V| \geq 1$. By (2.2), (3.15) and (3.16), we have

$$
\begin{aligned}
\mid T Q \| & (T Q+1) \xi-(T P+1) \mid \\
& =\left|\frac{(T Q+1)}{Q_{n}}\left(\frac{(T Q+1) \xi-(T P+1)}{Q_{n-1} \xi-P_{n-1}}\right)\right| \\
& =\left|U \frac{Q_{n-1}}{Q_{n}}+V\right|\left|U+V \frac{Q_{n} \xi-P_{n}}{Q_{n-1} \xi-P_{n-1}}\right| \\
& =\left|U V\left(1+\frac{Q_{n-1}\left(Q_{n} \xi-P_{n}\right)}{Q_{n}\left(Q_{n-1} \xi-P_{n-1}\right)}\right)+U^{2} \frac{Q_{n-1}}{Q_{n}}+V^{2} \frac{Q_{n} \xi-P_{n}}{Q_{n-1} \xi-P_{n-1}}\right| .
\end{aligned}
$$

The first term in the last expression has absolute value at least 1 as we argued $\left|Q_{n+1}\right|>\left|Q_{n}\right|>\left|Q_{n-1}\right|$ before. The summands involving $U^{2}$ and $V^{2}$ have strictly smaller norm because of (2.2), (2.4), (3.17) and (3.18). We thus have a contradiction to the hypothesis in (3.14).

The following statement, whose proof is omitted, also implies that our bound in Minkowski's theorem is the best possible.

Proposition 3.4. Let $\xi=\left[A_{1}, A_{2}, \cdots\right] \in T^{-1} \mathbb{F}_{q}\left[\left[T^{-1}\right]\right] \backslash K$ be such that there is a non-constant, irreducible polynomial $R$ which divides $A_{i}$ in $\mathbb{F}_{q}[T]$ for all $i>0$. Then, there exists an $\alpha \in K_{\infty}$ such that

$$
\|Q \xi-\alpha\| \geq|R|^{-2}|Q|^{-1}
$$

for all $Q \in \mathbb{F}_{q}[T] \backslash\{0\}$.

In this theorem, it implies that $\omega(\xi, \alpha)=1$ for such a $(\xi, \alpha)$. Actually, this is also the value of the asymptotic exponent for any $\xi \in K_{\infty} \backslash K$ and almost all $\alpha$. Let us first observe:

Proposition 3.5. For any irrational element $\xi$ in $K_{\infty}$, we have $\widehat{\omega}(\xi)=1$.

Proof. We denote the (infinite) sequence of convergents to $\xi$ by $\left(P_{k} / Q_{k}\right)_{k \geq 1}$ as before. For $k$ sufficiently large, let $Q$ be any non-zero polynomial with $|Q| \leq$ $q^{-1}\left|Q_{k}\right|=: H_{k}$. Because the convergents are well-known to be the best approximants of second kind, it follows that

$$
\|Q \xi\| \geq\left\|Q_{k-1} \xi\right\|=\frac{1}{\left|Q_{k}\right|}=\frac{1}{q H_{k}}
$$

This shows that $\widehat{\omega}(\xi)$ can be at most equal to 1 . The fact that it is equal to 1 is then obvious from Dirichlet's Theorem.

After this, we invoke Theorem 1.2 of [2] which states

Theorem 3.6. Let $A \in M_{m \times n}\left(K_{\infty}\right)$ and $\underline{\theta} \in K_{\infty}^{m}$. Then,

$$
\omega(A, \underline{\theta}) \geq \frac{1}{\widehat{\omega}\left({ }^{t} A\right)} \quad \text { and } \quad \widehat{\omega}(A, \underline{\theta}) \geq \frac{1}{\omega\left({ }^{t} A\right)}
$$

with both inequalities actually being equalities for (Haar-) almost all $\underline{\theta} \in K_{\infty}^{m}$. 
Our next endeavour is to prove that there is no uniform positive lower bound for the function $\widehat{\omega}(\xi, \alpha)$. The proposition below is in the spirit of Theorem III of [3, Chap. 3] and our proof strategy borrows heavily from theirs.

Proposition 3.7. Let $\Psi: \mathbb{N} \rightarrow \mathbb{R}_{>0}$ be an approximating function with $\Psi(x) \rightarrow 0$ as $x \rightarrow \infty$. Then, there exists a pair $(\xi, \alpha) \in K_{\infty}^{2}$ such that the system

$$
\|Q \xi-\alpha\| \leq \Psi(H), \quad|Q| \leq H
$$

does not have a solution for infinitely many $H$.

Proof. We fix $\alpha=T^{-1}$ and our desired element $\xi$ shall be the limit of a sequence of rational functions $R_{n} / S_{n}, n \in \mathbb{N}$, where $S_{n} \in \mathbb{F}_{q}[T] \backslash T \mathbb{F}_{q}[T]$ for all $n$. We note that $\left\|Q \frac{R_{n}}{S_{n}}-\alpha\right\| \geq\left(q\left|S_{n}\right|\right)^{-1}$ for all elements of this sequence and any $Q \in \mathbb{F}_{q}[T]$. Let $R_{0} / S_{0}=0$ and $R_{1} / S_{1}=(T+1)^{-1}$. In parallel, we construct a sequence $\left\{H_{n}\right\}_{n \geq 2} \subset \mathbb{N}$ as follows:

Assuming that $R_{n}, S_{n}, H_{n}$ have been defined for all $n \leq N$, let $H_{N+1}$ be the smallest for which

$$
\begin{aligned}
\Psi\left(H_{N+1}\right) & <\frac{1}{q^{2}\left|S_{N}\right|} \quad(N \geq 1), \text { and } \\
H_{N+1} & >H_{N} \quad(N>1) .
\end{aligned}
$$

As $\Psi(x) \rightarrow 0$ at infinity, such a number can be found. Now, choose any $S_{N+1}$ with a non-zero constant term and $\left|S_{N+1}\right| \geq q H_{N+1}\left|S_{N}\right|$ and let $R_{N+1}$ equal the integral part $\left[S_{N+1} R_{N} S_{N}^{-1}\right]$. Then,

$$
\left|\frac{R_{N+1}}{S_{N+1}}-\frac{R_{N}}{S_{N}}\right| \leq \frac{1}{q\left|S_{N+1}\right|} \leq \frac{1}{q^{2}\left|S_{N}\right| H_{N+1}}
$$

and the limit $\xi:=\lim _{n \rightarrow \infty} R_{n} / S_{n}=\lim _{n \rightarrow \infty} \sum_{i=1}^{n}\left(R_{i} / S_{i}-R_{i-1} / S_{i-1}\right)$ exists. Moreover,

$$
\left|\xi-\frac{R_{n}}{S_{n}}\right|=\lim _{m \rightarrow \infty}\left|\frac{R_{n+m}}{S_{n+m}}-\frac{R_{n}}{S_{n}}\right| \leq \frac{1}{q^{2}\left|S_{n}\right| H_{n+1}}
$$

because of the ultrametric inequality. If now $Q \in \mathbb{F}_{q}[T]$ with $|Q| \leq H_{n+1}$ and $P \in \mathbb{F}_{q}[T]$ be any polynomial,

$$
\begin{aligned}
|Q \xi-\alpha-P| & \geq\left|Q \frac{R_{n}}{S_{n}}-\alpha-P\right|-|Q|\left|\xi-\frac{R_{n}}{S_{n}}\right| \\
& \geq\left\|Q \frac{R_{n}}{S_{n}}-\alpha\right\|-\frac{1}{q^{2}\left|S_{n}\right|} \\
& \geq \frac{1}{q\left|S_{n}\right|}-\frac{1}{q^{2}\left|S_{n}\right|} \geq \frac{1}{q^{2}\left|S_{n}\right|}>\Psi\left(H_{n+1}\right)
\end{aligned}
$$

using (3.21) and (3.23). Since this is true for any $P$, the sequence $\left\{H_{n}\right\}$ constitutes the required set of infinitely many insoluble cases.

The following result is also implied by Theorem 2.3 of [2]. Here, we give another simple proof.

Corollary 3.8. There exists $(\xi, \alpha)$ such that $\widehat{\omega}(\xi, \alpha)=0$. 
Proof. Let $\Psi(n):=n^{-1 / \log \log n}$. It clearly goes to zero as $n$ tends to $\infty$. In addition for any $\varepsilon>0, \Psi(n)>n^{-\varepsilon}$ eventually and hence, the pair $(\xi, \alpha)$ corresponding to $\Psi$ given to us by Prop. 3.7 has $\widehat{\omega}(\xi, \alpha) \leq \varepsilon$. Our choice of $\varepsilon$ was arbitrary.

We will like to end this section with a brief discussion on the issue of monicity. This is to say that our concern is to find solutions to the inhomogeneous inequality

$$
|Q \xi-\alpha-P|<\varepsilon
$$

when the polynomial $Q$ is restricted to be monic and $\xi \notin K$. The argument given below follows closely that of Kronecker for real numbers.

Proof of Proposition 1.2. From the function field Dirichlet's theorem, one knows that there are infinitely many polynomial pairs $(R, S)$ for which

$$
S \xi-R=\xi_{1} / S
$$

with $\left|\xi_{1}\right| \leq 1 / q$ and $\operatorname{gcd}(R, S)=1$. We may assume here that $S$ is monic because homogeneity. The required polynomials for the inhomogeneous problem may be obtained by perturbing each of these $S$ where the amount of perturbation is determined by $\alpha$. Consider the polynomial part $[S \alpha]$. As $R$ and $S$ are co-prime in $\mathbb{F}_{q}[T]$, we will be able to find polynomials $P_{1}$ and $P_{2}$ such that $\left|P_{1}\right|<|S|$ and

$$
[S \alpha]=R P_{1}+S P_{2} \text {. }
$$

Then, we have

$$
S\left(P_{1} \xi+P_{2}\right)=R P_{1}+S P_{2}+\xi_{1} / S=S \alpha+\alpha_{1}+\xi_{1} / S
$$

where again $\left|\alpha_{1}\right| \leq 1 / q$. On rearranging,

$$
\left|P_{1} \xi-\alpha+P_{2}\right| \leq \frac{1}{q|S|} .
$$

This bound does not change if we add the term $S \xi-R$ to the left-side expression. Furthermore, the polynomial $S+P_{1}$ is monic as well as $\left|S+P_{1}\right|=|S|$.

The above proposition implies that the set $\left\{\langle Q \xi\rangle \mid Q \in \mathbb{F}_{q}[T]\right.$, monic $\}$ is also dense in $T^{-1} \mathbb{F}_{q}\left[\left[T^{-1}\right]\right]$. We can moreover derive a corollary from this result. Given $\varepsilon>0$, choose any monic $\eta \in K_{\infty} \backslash K(\xi, \alpha)$ with $|\eta|<\varepsilon$ and replace $\alpha$ by $\alpha+\eta$ in Proposition 1.2. The infinitely many monic solutions in $Q$ whose norm is more than $1 / \varepsilon$ will all help us to realize the constrained inequality

$$
|Q \xi-\alpha-P|<\varepsilon
$$

under the demand that $Q$ and $Q \xi-\alpha-P$ be monic.

\section{Exponents For $\mathrm{SL}_{2}\left(\mathbb{F}_{q}[T]\right)$ ACTION}

Without any loss of generality, we assume that the starting point $\underline{x}$ equals ${ }^{t}(\xi, 1)$. If needed, we can also use the matrix

$$
J:=\left(\begin{array}{cc}
0 & -1 \\
1 & 0
\end{array}\right)
$$

to have $|\xi| \leq 1$. This will mean that $\left|P_{k}\right| \leq\left|Q_{k}\right|$ for all $k \geq 0$, where $P_{k} / Q_{k}$ is the $k$-th convergent to $\xi$. Being a (signed) permutation matrix, $J$ has the desirable property that $|J \underline{x}|=|\underline{x}|$ for all $\underline{x}$ in $K_{\infty}^{2}$ as well as $|J \gamma|=|\gamma J|=|\gamma|$ for all $2 \times 2$ matrices $\gamma$. 
Lemma 4.1. Let $\gamma \in \mathrm{SL}_{2}\left(\mathbb{F}_{q}[T]\right)$ be such that $|\gamma|<\left|Q_{k+1}\right|$ where $P_{k} / Q_{k}$ are convergents to $\xi$. Then,

$$
|\gamma \underline{x}| \geq \frac{1}{\left|Q_{k}\right|}
$$

The argument here is same as the one used in [10, 16] except that we get tighter bounds owing to the ultrametric inequality. Now if $k$ is chosen so as to have $\left|Q_{k}\right| \leq|\gamma|<\left|Q_{k+1}\right|$, we immediately get $\mu(\underline{x}, \underline{0}) \leq 1$. At the same time,

$$
M_{k} \underline{x}=\left(\begin{array}{c}
Q_{k} \xi-P_{k} \\
(-1)^{k-1}\left(Q_{k-1} \xi-P_{k-1}\right)
\end{array}\right)
$$

for

$$
M_{k}:=\left(\begin{array}{cc}
Q_{k} & -P_{k} \\
(-1)^{k-1} Q_{k-1} & (-1)^{k} P_{k-1}
\end{array}\right)
$$

and therefore, $\left|M_{k} \underline{x}\right|=\left|\varepsilon_{k-1}\right|=1 /\left|Q_{k}\right|$ for all $k \in \mathbb{N}$ where $\varepsilon_{n}:=Q_{n} \xi-P_{n}$ is the scaled error (with sign) for approximation by the $n$-th convergent of $\xi$. By our assumption that $|\xi| \leq 1$, we get $\left|P_{k-1}\right| \leq\left|Q_{k-1}\right|,\left|P_{k}\right| \leq\left|Q_{k}\right|$ and $\left|Q_{k-1}\right|<$ $\left|Q_{k}\right|=\left|M_{k}\right|$. Thereby, one has $\left|M_{k} \underline{x}\right|=1 /\left|M_{k}\right|$. As $k \rightarrow \infty$, we get infinitely many such matrices in $\mathrm{SL}_{2}\left(\mathbb{F}_{q}[T]\right)$. The conclusion is

$$
\mu(\underline{x}, \underline{0})=1 .
$$

This also gives us an upper bound for the uniform exponent $\widehat{\mu}(\underline{x}, \underline{0})$. We can actually improve upon this. If $\omega<\omega(\xi)$, it follows from the definition of $\omega(\xi)$ that $\left|Q_{k+1}\right| \geq\left|Q_{k}\right|^{\omega}$ for infinitely many $k$. Consider $H=\left|Q_{k+1}\right| / q$ for such a $k$ so that for all matrices $\gamma \in \mathrm{SL}_{2}\left(\mathbb{F}_{q}[T]\right)$ with $|\gamma| \leq H$,

$$
|\gamma \underline{x}| \geq \frac{1}{\left|Q_{k}\right|} \geq \frac{1}{\left|Q_{k+1}\right|^{1 / \omega}}=\frac{1}{(q H)^{1 / \omega}}
$$

by Lemma 4.1. Said differently, $\widehat{\mu}(\underline{x}, \underline{0})$ can be at most $1 / \omega$. This is strengthened to $1 / \omega(\xi)$ by letting $\omega \rightarrow \omega(\xi)$ from below. For the lower bound, let $T \gg 1$ be so that there exists some $k \in \mathbb{N}$ for which $\left|Q_{k}\right|=\left|M_{k}\right| \leq T<\left|M_{k+1}\right|=\left|Q_{k+1}\right|$. We then have

$$
\left|M_{k} \underline{x}\right|=\frac{1}{\left|Q_{k}\right|}<\frac{1}{\left|Q_{k+1}\right|^{1 / \omega}}<\frac{1}{T^{1 / \omega}}
$$

for all $k \gg 1$ and $\omega>\omega(\xi)$ from the discussion in $\S 2$. Let $\omega$ approach $\omega(\xi)$ from the right and we can write down

Proposition 4.2. Let $\underline{x}={ }^{t}\left(x_{1}, x_{2}\right) \in K_{\infty}^{2}$ such that $\xi=x_{1} / x_{2}$ is not in $K$. Then,

$$
\mu(\underline{x}, \underline{0})=1 \quad \text { and } \quad \widehat{\mu}(\underline{x}, \underline{0})=1 / \omega(\xi) .
$$

From (1.4), we see that the two exponents are equal for almost all $\underline{x}$ with respect to the Haar measure. Our next goal is to bound the size of an $\mathrm{SL}_{2}\left(\overline{\mathbb{F}}_{q}[T]\right)$ matrix in terms of convergent and upper-triangular matrices. In the sequel, for $a$ in $\mathbb{F}_{q}[T]$, we set

$$
U(a)=\left(\begin{array}{ll}
1 & a \\
0 & 1
\end{array}\right)
$$


Lemma 4.3 (cf. [10]). Let $k \in \mathbb{N}$ and

$$
N=\left(\begin{array}{cc}
t & t^{\prime} \\
s & s^{\prime}
\end{array}\right)
$$

be any matrix in $\mathrm{SL}_{2}\left(\mathbb{F}_{q}[T]\right)$. Then, the product $\gamma=N U(a) M_{k}$ satisfies

$$
\left|\left(a Q_{k-1}+(-1)^{k-1} Q_{k}\right) s\right|-\left|s^{\prime} Q_{k-1}\right| \leq|\gamma| \leq|N| \cdot \max \left\{\left|Q_{k}\right|,\left|a Q_{k-1}\right|\right\} .
$$

Proof. As we have seen before in [10] and [16],

$$
\begin{aligned}
\gamma & =\left(\begin{array}{ll}
t & t^{\prime} \\
s & s^{\prime}
\end{array}\right)\left(\begin{array}{ll}
1 & a \\
0 & 1
\end{array}\right)\left(\begin{array}{cc}
Q_{k} & -P_{k} \\
(-1)^{k-1} Q_{k-1} & (-1)^{k} P_{k-1}
\end{array}\right) \\
& =\left(\begin{array}{cc}
t Q_{k}+(-1)^{k-1} Q_{k-1}\left(t a+t^{\prime}\right) & -t P_{k}+(-1)^{k} P_{k-1}\left(t a+t^{\prime}\right) \\
s Q_{k}+(-1)^{k-1} Q_{k-1}\left(s a+s^{\prime}\right) & -s P_{k}+(-1)^{k} P_{k-1}\left(s a+s^{\prime}\right)
\end{array}\right) .
\end{aligned}
$$

The lower bound is given to us by the bottom left entry of this last matrix. Since $|\xi|$ was assumed to be at most 1 and irrational, $\left|P_{n}\right| \leq\left|Q_{n}\right|<\left|Q_{n+1}\right|$ for all $n \in \mathbb{N}$. This leads to the upper bound.

Being done with that, we now want an upper bound on the size of the vector $\gamma \underline{x}$ itself. The statement below is the function field analogue of [10, Lemma 3].

Lemma 4.4. Let $a, k, N$ and $\gamma=N U(a) M_{k}=:\left(\begin{array}{ll}V_{1} & U_{1} \\ V_{2} & U_{2}\end{array}\right)$ be as in Lemma 4.3,

For $y \in K_{\infty}$, defining $\delta=s y-t$ and $\delta^{\prime}=s^{\prime} y-t^{\prime}$ gives us

$$
\left|V_{1} \xi+U_{1}-y\left(V_{2} \xi+U_{2}\right)\right| \leq \max \left\{\left|\frac{\delta}{Q_{k+1}}\right|,\left|\frac{\delta a+\delta^{\prime}}{Q_{k}}\right|\right\} .
$$

Proof. We have

$$
\begin{aligned}
y\left(V_{2} \xi+U_{2}\right)-\left(V_{1} \xi+U_{1}\right) & =\left(\begin{array}{ll}
-1 & y
\end{array}\right) \gamma\left(\begin{array}{l}
\xi \\
1
\end{array}\right)=\left(\begin{array}{ll}
-1 & y
\end{array}\right)\left(\begin{array}{ll}
t & t^{\prime} \\
s & s^{\prime}
\end{array}\right) U(a) M_{k}\left(\begin{array}{l}
\xi \\
1
\end{array}\right) \\
& =\left(\begin{array}{ll}
\delta & \delta^{\prime}
\end{array}\right) U(a)\left(\begin{array}{c}
\varepsilon_{k} \\
(-1)^{k-1} \varepsilon_{k-1}
\end{array}\right) \\
& =\delta \varepsilon_{k}+(-1)^{k-1}\left(\delta a+\delta^{\prime}\right) \varepsilon_{k-1} .
\end{aligned}
$$

In order to finish the argument, one only needs to use $\left|\varepsilon_{n}\right|=1 /\left|Q_{n+1}\right|$ for all $n \in \mathbb{N}$ and the ultrametric property.

Consider any vector $\underline{y}={ }^{t}\left(y_{1}, y_{2}\right) \in K_{\infty}^{2}$ and let ${ }^{t}\left(\Lambda_{1}, \Lambda_{2}\right)$ denote the difference $\gamma \underline{x}-\underline{y}$, i. e.,

$$
\Lambda_{i}=x_{2}\left(V_{i} \xi+U_{i}\right)-y_{i} \text { for } i \in\{1,2\},
$$

where we take $\underline{x}={ }^{t}\left(x_{1}, x_{2}\right)$ such that $|\underline{x}|=\left|x_{2}\right|>0$ without loss of generality. In particular, when we choose $y$ to be the slope $y_{1} / y_{2}$ of our target $y$ (again assume $|y| \leq 1$ using the matrix $J$ ), Lemma 4.4 tells us that

$$
\begin{aligned}
\left|\Lambda_{1}-y \Lambda_{2}\right| & =\left|x_{2}\left(\left(V_{1} \xi+U_{1}\right)-y\left(V_{2} \xi+U_{2}\right)\right)\right| \\
& \leq\left|x_{2}\right| \cdot \max \left\{\left|\frac{\delta}{Q_{k+1}}\right|,\left|\frac{\delta a+\delta^{\prime}}{Q_{k}}\right|\right\} .
\end{aligned}
$$


INHOMOGENEOUS DIOPHANTINE APPROXIMATION OVER POWER SERIES FIELDS 13

The idea is simple. To get a bound on the size of ${ }^{t}\left(\Lambda_{1}, \Lambda_{2}\right)$, we separately bound each of the component $\Lambda_{2}$ and the quantity $\left|\Lambda_{1}-y \Lambda_{2}\right|$. From (4.10), we deduce that

$$
\begin{aligned}
\Lambda_{2} & =x_{2}\left(V_{2} \xi+U_{2}\right)-y_{2}=x_{2}\left(s \varepsilon_{k}+(-1)^{k-1}\left(s a+s^{\prime}\right) \varepsilon_{k-1}\right)-y_{2} \\
& =(-1)^{k-1} x_{2} s \varepsilon_{k-1}(a-\rho),
\end{aligned}
$$

where

$$
\rho:=\frac{(-1)^{k-1} y_{2}}{x_{2} s \varepsilon_{k-1}}-\frac{(-1)^{k-1} \varepsilon_{k}}{\varepsilon_{k-1}}-\frac{s^{\prime}}{s} .
$$

The element $\rho \in K_{\infty}$ is the one which decides the value of $a$ for us, namely we take $a=[\rho]$ so that $|a-\rho| \leq 1 / q$ and $|a| \leq|\rho|$. Such a choice means $\left|\Lambda_{2}\right|<\left|x_{2} s / Q_{k}\right|$.

4.1. Target points with slope in $K$. Let $\underline{y}$ have slope $y=y_{1} / y_{2}=A / B \in K$ such that $A, B \in \mathbb{F}_{q}[T], \operatorname{gcd}\{A, B\} \in \mathbb{F}_{q}^{*}$ and $|A / B| \leq 1$. Now, assign

$$
N=\left(\begin{array}{cc}
A & \widetilde{A} \\
B & \widetilde{B}
\end{array}\right)
$$

where $\widetilde{A}$ and $\widetilde{B}$ are (upto multiplication by \pm 1 ) respectively the numerators and denominators of the penultimate convergent in the continued fraction expansion of $A / B$. This ensures that $\operatorname{det} N=1$ always and $|\widetilde{A}| \leq|\widetilde{B}|<|B|=|N|$ except when $\widetilde{B}=0$ and $A, \widetilde{A}, B \in \mathbb{F}_{q}^{*}$ giving $|N|=|B|=1$ again.

Lemma 4.5 (cf. [10, Lemma 5]). Let $k \gg 1$ and $y \in K_{\infty}^{2}$ with slope $y=A / B \in K$. Then, there exists $\gamma=N U(a) M_{k} \in \mathrm{SL}_{2}\left(\mathbb{F}_{q}[T]\right)$ satisfying

$$
|\gamma|=\left|\frac{y_{2}}{x_{2}}\right|\left|Q_{k} Q_{k-1}\right|<\left|\frac{y_{2}}{x_{2}}\right|\left|Q_{k}\right|^{2} \text { and }|\gamma \underline{x}-\underline{y}| \leq\left|\frac{B x_{2}}{Q_{k}}\right| .
$$

Proof. In this case, $\delta=B y-A=0$ and $\delta^{\prime}=\widetilde{B} y-\widetilde{A}=1 / B$ so that

$$
\left|\Lambda_{1}-y \Lambda_{2}\right| \leq \frac{\left|x_{2}\right|}{\left|B Q_{k}\right|} \text { and }\left|\Lambda_{2}\right| \leq \frac{\left|x_{2} B\right|}{q\left|Q_{k}\right|} .
$$

After this, the ultrametric inequality gives the upper bound for $|\gamma \underline{x}-\underline{y}|$. Next,

$$
|a|=|\rho|=\left|\frac{y_{2} Q_{k}}{x_{2} B}\right|>1
$$

for all $k$ large enough as $\left|\varepsilon_{k} / \varepsilon_{k-1}\right|,|\widetilde{B} / B|<1$ whereas the norm of the first term on the right side of (4.14) increases with $k$. We can now improve upon Lemma 4.3 to have more precise knowledge about the size of $\gamma$.

For any such matrix $\gamma$, one thus gets

$$
|\gamma \underline{x}-\underline{y}| \leq\left|\frac{B x_{2}}{Q_{k}}\right|<|B|\left|x_{2} y_{2}\right|^{1 / 2}|\gamma|^{-1 / 2} .
$$

We should, however, be more careful when discussing the asymptotic exponent $\mu(\underline{x}, \underline{y})$. Let $\omega<\omega(\xi)$ so that $\left|Q_{k-1}\right| \leq\left|Q_{k}\right|^{1 / \omega}$ for infinitely many $k$ from the observations following (2.7). Then, $|\gamma| \ll_{\underline{x}, \underline{y}}\left|Q_{k}\right|^{1+\frac{1}{\omega}}$ and $|\gamma \underline{x}-\underline{y}| \ll|\gamma|^{-\frac{\omega}{\omega+1}}$ 
for all such $k$ 's. This implies that $\mu(\underline{x}, \underline{y}) \geq \omega /(\omega+1)$ firstly and since $\omega$ can be taken arbitrarily close to $\omega(\xi)$, we have

$$
\mu(\underline{x}, \underline{y}) \geq \frac{\omega(\xi)}{\omega(\xi)+1}
$$

as in the real case [10, $§ \S 6.2]$.

Let us move ahead to obtain a similar bound for $\widehat{\mu}(\underline{x}, \underline{y})$. Our claim is that it is at least $1 /(\omega(\xi)+1)$. The statement is trivial for $\omega(\xi)=\infty$, thus, we assume that $\omega(\xi)$ is finite. For any $T \gg 1$, there is a unique index $k$ for which

$$
\left|\frac{y_{2}}{x_{2}}\right|\left|Q_{k-1} Q_{k}\right| \leq T<\left|\frac{y_{2}}{x_{2}}\right|\left|Q_{k} Q_{k+1}\right| .
$$

Then, $T<\left|Q_{k}\right|^{\omega+1}$ for given $\omega>\omega(\xi)$ and all $k$ large enough, while Lemma 4.5tells us that there exists a $\gamma=N U(a) M_{k} \in \mathrm{SL}_{2}\left(\mathbb{F}_{q}[T]\right)$ with $|\gamma| \leq T$ and $|\gamma \underline{x}-\underline{y}| \leq$ $\left|B x_{2}\right| T^{-1 /(\omega+1)}$. Since this is true for all $T$ large enough and $\omega$ was arbitrary, we conclude that

$$
\widehat{\mu}(\underline{x}, \underline{y}) \geq \frac{1}{\omega(\xi)+1},
$$

where $\xi=x_{1} / x_{2}$ is the slope of the starting point $\underline{x}$.

Our next goal is to show that the inequalities (4.19) and (4.21) are actually equalities when the target $\underline{y}$ has slope in $K$. We start with $\mu(\underline{x}, \underline{y})$. The trick is to break down any matrix $\gamma$ using the various convergent matrices for $\xi$ and $y$, which are already familiar to us from (4.3) (see also [10, Theorem 4]). The theorem given below is an inhomogeneous version of Lemma 4.1 for rational target points.

Theorem 4.6. Let $\underline{x}, \underline{y} \in K_{\infty}^{2}$ be such that $x_{1} / x_{2} \in K_{\infty} \backslash K$ and $y_{1} / y_{2} \in K$. For any $k$ such that $\left|Q_{k}\right|>\left|B x_{2} / y_{2}\right|$ and $\gamma \in \mathrm{SL}_{2}\left(\mathbb{F}_{q}[T]\right)$ with $|\gamma|<\left|y_{2} Q_{k} Q_{k+1} / x_{2}\right|$, we must have an approximation error $|\gamma \underline{x}-\underline{y}| \geq\left|x_{2} /\left(B Q_{k}\right)\right|$.

Proof. Let us assume that $\gamma=\left(\begin{array}{cc}V_{1} & U_{1} \\ V_{2} & U_{2}\end{array}\right)$ is such that ${ }^{t}\left(\Lambda_{1}, \Lambda_{2}\right)=\gamma \underline{x}-\underline{y}$ satisfies $|\gamma \underline{x}-\underline{y}|<\left|x_{2} /\left(B Q_{k}\right)\right|$ and we will reach a contradiction. Denote

$$
\begin{aligned}
\widetilde{\gamma} & =\left(\begin{array}{cc}
\widetilde{V}_{1} & \widetilde{U}_{1} \\
\widetilde{V}_{2} & \widetilde{U}_{2}
\end{array}\right):=N^{-1} \gamma \\
& =\left(\begin{array}{cc}
\frac{\widetilde{B}\left(V_{1} y_{2}-V_{2} y_{1}\right)}{y_{2}}+\frac{V_{2}}{B} & \frac{\widetilde{B}\left(U_{1} y_{2}-U_{2} y_{1}\right)}{y_{2}}+\frac{U_{2}}{B} \\
-\frac{B\left(V_{1} y_{2}-V_{2} y_{1}\right)}{y_{2}} & -\frac{B\left(U_{1} y_{2}-U_{2} y_{1}\right)}{y_{2}}
\end{array}\right)
\end{aligned}
$$

so that

$$
\widetilde{\gamma} \underline{x}=x_{2}\left[\begin{array}{c}
\widetilde{V}_{1} \xi+\widetilde{U}_{1} \\
\widetilde{V}_{2} \xi+\widetilde{U}_{2}
\end{array}\right]=N^{-1}\left(\underline{y}+\left[\begin{array}{c}
\Lambda_{1} \\
\Lambda_{2}
\end{array}\right]\right)=\left[\begin{array}{c}
\frac{y_{2}}{B}+\widetilde{B} \Lambda_{1}-\widetilde{A} \Lambda_{2} \\
-B \Lambda_{1}+A \Lambda_{2}
\end{array}\right]
$$

by virtue of the fact that $\widetilde{B} y_{1}-\widetilde{A} y_{2}$ equals $y_{2} / B$. Then, the determinant

$$
\left|\begin{array}{ll}
V_{1} & y_{1} \\
V_{2} & y_{2}
\end{array}\right|=\left|\begin{array}{ll}
V_{1} & \left(V_{1} \xi+U_{1}\right) x_{2}-\Lambda_{1} \\
V_{2} & \left(V_{2} \xi+U_{2}\right) x_{2}-\Lambda_{2}
\end{array}\right|=x_{2}-\left|\begin{array}{cc}
V_{1} & \Lambda_{1} \\
V_{2} & \Lambda_{2}
\end{array}\right|
$$


and its norm satisfies

$$
\left|V_{1} y_{2}-V_{2} y_{1}\right| \leq \max \left\{\left|x_{2}\right|,|\gamma| \cdot|\gamma \underline{x}-\underline{y}|\right\} \leq \max \left\{\left|x_{2}\right|,\left|y_{2} Q_{k+1} / B\right|\right\} .
$$

The second of these terms in the upper bound will clearly dominate when $\left|Q_{k}\right|>$ $\left|B x_{2} / y_{2}\right|$. Because of our supposition, one gets for all such $k$ that

$$
\begin{aligned}
\left|\widetilde{V}_{2}\right| & =\left|\frac{B}{y_{2}}\left(V_{1} y_{2}-V_{2} y_{1}\right)\right|<\left|Q_{k+1}\right|, \text { and } \\
\left|\widetilde{V}_{2} \xi+\widetilde{U}_{2}\right| & =\frac{1}{\left|x_{2}\right|}\left|B \Lambda_{1}-A \Lambda_{2}\right| \leq\left|\frac{B}{x_{2}}\right||\gamma \underline{x}-\underline{y}|<\frac{1}{\left|Q_{k}\right|} .
\end{aligned}
$$

We are now ready to consider the matrix $N^{-1} \gamma M_{k}^{-1}=\widetilde{\gamma} M_{k}^{-1}$ and more specifically, tackle its lower right entry given by $\widetilde{V}_{2} P_{k}+Q_{k} \widetilde{U}_{2}$. Its size is bounded as follows:

$$
\left|\widetilde{V}_{2} P_{k}+Q_{k} \widetilde{U}_{2}\right|=\left|-\widetilde{V}_{2}\left(Q_{k} \xi-P_{k}\right)+Q_{k}\left(\widetilde{V}_{2} \xi+\widetilde{U}_{2}\right)\right|<1
$$

Since all the three matrices $N, \gamma$ and $M_{k}$ have polynomial entries and determinant 1 , the entry in (4.27) must be zero. Consequently, $G:=N^{-1} \gamma M_{k}^{-1}$ is of the form

$$
\left(\begin{array}{cc}
R & \zeta \\
-\zeta^{-1} & 0
\end{array}\right)
$$

for some $R \in \mathbb{F}_{q}[T]$ and $\zeta \in \mathbb{F}_{q}^{*}$ but that would mean

$$
\tilde{\gamma} \underline{x}=G M_{k} \underline{x}=x_{2}\left[\begin{array}{c}
R \varepsilon_{k}+(-1)^{k-1} \zeta \varepsilon_{k-1} \\
-\zeta^{-1} \varepsilon_{k}
\end{array}\right]=\left[\begin{array}{c}
\frac{y_{2}}{B}+\widetilde{B} \Lambda_{1}-\widetilde{A} \Lambda_{2} \\
-B \Lambda_{1}+A \Lambda_{2}
\end{array}\right] .
$$

Let us focus on the second coordinate of this column vector. In the last representation, $\left|B \Lambda_{1}-A \Lambda_{2}\right|<|B||\gamma \underline{x}-\underline{y}|<\left|x_{2} / Q_{k}\right|<\left|y_{2} / B\right|$ owing to our hypothesis about $k$. Thus, $\widetilde{\gamma} \underline{x}$ has norm equal to $\left|y_{2} / B\right|$ and this in turn forces $|R|=\left|y_{2} Q_{k+1} /\left(x_{2} B\right)\right|$. We use this knowledge to get a lower bound for $|\gamma|$ as

$$
\gamma=N G M_{k}=\left(\begin{array}{cc}
* & * \\
B R Q_{k}+(-1)^{k-1} \zeta B Q_{k-1}-\zeta^{-1} \widetilde{B} Q_{k} & *
\end{array}\right)
$$

implies that the leftmost summand of the lower left entry rises much faster in size with $k$ than the other two terms whence $|\gamma| \geq\left|y_{2} Q_{k} Q_{k+1} / x_{2}\right|$, which is a contradiction. The conclusion is that $|\gamma \underline{x}-\underline{y}|$ has to be at least $\left|x_{2} /\left(B Q_{k}\right)\right|$.

Given any $\gamma \in \mathrm{SL}_{2}\left(\mathbb{F}_{q}[T]\right)$ of sufficiently large norm, we can find a unique $k$ such that

$$
\left|\frac{y_{2}}{x_{2}}\right|\left|Q_{k-1} Q_{k}\right| \leq|\gamma|<\left|\frac{y_{2}}{x_{2}}\right|\left|Q_{k} Q_{k+1}\right| .
$$

If $\omega(\xi)$ is finite, choose any $\omega>\omega(\xi)$ so that $\left|Q_{k-1}\right| \geq\left|Q_{k}\right|^{1 / \omega}$ eventually. Then, Theorem 4.6 tells us

$$
|\gamma \underline{x}-\underline{y}| \geq \frac{\left|x_{2}\right|}{\left|B Q_{k}\right|} \gg_{\underline{x}, \underline{y}} \frac{1}{|\gamma|^{\omega /(\omega+1)}}
$$

for all such $\gamma$ and on letting $\omega$ tend to $\omega(\xi)$, we have

$$
\mu(\underline{x}, \underline{y}) \leq \frac{\omega(\xi)}{\omega(\xi)+1} .
$$

When $\omega(\xi)=\infty$, we instead argue that $|\gamma \underline{x}-\underline{y}| \gg_{\underline{x}, \underline{y}} 1 /|\gamma|$ so that $\mu(\underline{x}, \underline{y}) \leq 1=$ $(1+1 / \omega(\xi))^{-1}$ again. 
For the uniform exponent, let us fix some $\omega<\omega(\xi)$ which means there are infinitely many denominators $\left|Q_{k+1}\right| \geq\left|Q_{k}\right|^{\omega}$. Now, consider the diverging subsequence $H_{k}:=q^{-1}\left|y_{2} Q_{k} Q_{k+1} / x_{2}\right|$ corresponding to these indices $k$. In this scenario, $H_{k} \geq q^{-1}\left|y_{2} / x_{2}\right|\left|Q_{k}\right|^{\omega+1}$ and Theorem 4.6] is saying

$$
|\gamma \underline{x}-\underline{y}| \gg_{\underline{x}, \underline{y}} \frac{1}{\left|Q_{k}\right|} \gg_{\underline{x}, \underline{y}} \frac{1}{H_{k}^{1 /(\omega+1)}}
$$

for all $\gamma$ with $|\gamma| \leq H_{k}$. This means that $\widehat{\mu}(\underline{x}, \underline{y})$ can be at most $1 /(\omega+1)$ and as our choice of $\omega<\omega(\xi)$ was arbitrary, we reach (2) of Theorem 1.4.

4.2. Target points with irrational slopes. Let us now start the last case when $\underline{y}={ }^{t}\left(y_{1}, y_{2}\right) \in K_{\infty}^{2} \backslash\{\underline{0}\}$ is such that $y=y_{1} / y_{2} \in K_{\infty} \backslash K$. We further take $|y| \leq 1$ using the matrix $J$. This constitutes the generic situation as far as the target points are concerned. If $R_{j-1} / S_{j-1}$ and $R_{j} / S_{j}$ are any consecutive continued fraction convergents to $y$, we take $N_{j}:=\left(\begin{array}{cc}R_{j} & \widetilde{R}_{j-1} \\ S_{j} & \widetilde{S}_{j-1}\end{array}\right) \in \mathrm{SL}_{2}\left(\mathbb{F}_{q}[T]\right)$, where

$$
\widetilde{R}_{j-1}=(-1)^{j-1} R_{j-1} \text { and } \widetilde{S}_{j-1}=(-1)^{j-1} S_{j-1} .
$$

Then, $\left|N_{j}\right|=\left|S_{j}\right|$ since that entry dominates all others and the term $\rho$ from (4.14) has absolute value

$$
\left|\frac{y_{2} Q_{k}}{x_{2} S_{j}}\right|-1<|\rho| \leq \max \left\{\left|\frac{y_{2} Q_{k}}{x_{2} S_{j}}\right|, 1\right\} .
$$

The polynomial part $a=[\rho]$ has the same norm as $\rho$ whenever $|\rho| \geq 1$ and equals 0 otherwise. Now, $|\rho| \geq 1$ if and only if $\left|y_{2} Q_{k} / x_{2} S_{j}\right| \geq 1$ and therefore, we are right to assert

$$
\left|\frac{y_{2} Q_{k}}{x_{2} S_{j}}\right|-1<|a| \leq\left|\frac{y_{2} Q_{k}}{x_{2} S_{j}}\right| .
$$

Lemma 4.7 (cf. [10, Lemma 4]). For all $j, k \in \mathbb{N}^{*}$ and $\underline{x}, \underline{y}$ as above, there exists $\gamma=N_{j} U(a) M_{k} \in \mathrm{SL}_{2}\left(\mathbb{F}_{q}[T]\right)$ for some $a \in \mathbb{F}_{q}[T]$ such that

$$
\begin{aligned}
& || \frac{y_{2} Q_{k} Q_{k-1}}{x_{2}}|-| S_{j} Q_{k}||-\left|S_{j-1} Q_{k-1}\right| \leq|\gamma| \leq \max \left\{\left|S_{j} Q_{k}\right|,\left|\frac{y_{2} Q_{k} Q_{k-1}}{x_{2}}\right|\right\} \\
& \text { and } \\
& \qquad|\gamma \underline{x}-\underline{y}| \leq \max \left\{\left|\frac{y_{2}}{S_{j} S_{j+1}}\right|,\left|\frac{x_{2} S_{j}}{Q_{k}}\right|\right\} .
\end{aligned}
$$

Proof. The quantities $\delta$ and $\delta^{\prime}$ introduced in the statement of Lemma 4.4 respectively equal $1 /\left|S_{j+1}\right|$ and $1 /\left|S_{j}\right|$ here. Also, the second component $\Lambda_{2}$ of our error vector $\gamma \underline{x}-\underline{y}$ is bounded above as

$$
\left|\Lambda_{2}\right|<\left|\frac{x_{2} S_{j}}{Q_{k}}\right|
$$

by (4.13). The reasoning for the first component is that firstly,

$$
\left|\Lambda_{1}\right| \leq \max \left\{\left|\Lambda_{1}-y \Lambda_{2}\right|,\left|y \Lambda_{2}\right|\right\} \leq \max \left\{\left|\Lambda_{1}-y \Lambda_{2}\right|,\left|\Lambda_{2}\right|\right\},
$$

while $\left|\Lambda_{1}-y \Lambda_{2}\right| \leq \max \left\{\left|y_{2}\right|\left|S_{j} S_{j+1}\right|^{-1},\left|x_{2}\right|\left|S_{j} Q_{k}\right|^{-1}\right\}$ from (4.12) and (4.37). Clearly, the term $\left|x_{2} S_{j} / Q_{k}\right|$ will matter more than $\left|x_{2} /\left(S_{j} Q_{k}\right)\right|$. 
The bounds mentioned for $\gamma$ follow from Lemma 4.3 and (4.37).

Proposition 4.8. For any pair consisting of a starting point $\underline{x} \in K_{\infty}^{2} \backslash\{\underline{0}\}$ and target $y$ with their respective slopes $\xi$ and $y$ irrational, one has

$$
\mu(\underline{x}, \underline{y}) \geq \frac{1}{3} \quad \text { and } \quad \widehat{\mu}(\underline{x}, \underline{y}) \geq \frac{\omega(y)+1}{2(2 \omega(y)+1) \omega(\xi)} \geq \frac{1}{4 \omega(\xi)}
$$

At this stage, we follow two parallel strategies as per whether $\omega(\xi)<3$ or $\omega(\xi)>2$.

4.2.1. The case $\omega(\xi)<3$. For any $j$ large enough, pick $k$ satisfying

$$
\left|\frac{y_{2} Q_{k-1}}{x_{2}}\right|^{1 / 3}<\left|S_{j}\right| \leq\left|\frac{y_{2} Q_{k}}{x_{2}}\right|^{1 / 3}<\left|S_{j+1}\right| .
$$

Let us substitute this into Lemma 4.7 which then gives

$$
|\gamma \underline{x}-\underline{y}|<\left|y_{2} x_{2}^{2}\right|^{1 / 3} \max \left\{\frac{1}{\left|Q_{k} Q_{k-1}\right|^{1 / 3}}, \frac{1}{\left|Q_{k}\right|^{2 / 3}}\right\}=\left|\frac{y_{2} x_{2}^{2}}{Q_{k} Q_{k-1}}\right|^{1 / 3} .
$$

Next, take some $\omega$ with $\omega(\xi)<\omega<3$ so that $\left|Q_{k-1}\right| \geq\left|Q_{k}\right|^{1 / \omega}>\left|Q_{k}\right|^{1 / 3}$ for all $k \gg 1$. On the other hand, we also have $\left|S_{j}\right| \ll_{\underline{x}, \underline{y}}\left|Q_{k}\right|^{1 / 3}$ by our construction which dictates that $|\gamma|=\left|y_{2} Q_{k} Q_{k-1} / x_{2}\right|$. Subsequently, one gets

$$
|\gamma \underline{x}-\underline{y}| \ll_{\underline{x}, \underline{y}}|\gamma|^{-1 / 3},
$$

for infinitely many such pairs $(j, k)$ and the infinite set of matrices $\left\{N_{j} U(a) M_{k}\right\} \subset$ $\mathrm{SL}_{2}\left(\mathbb{F}_{q}[T]\right)$ determined by them. In particular, we have shown that $\mu(\underline{x}, \underline{y}) \geq 1 / 3$.

4.2.2. The case $\omega(\xi)>2$. Fix any $\omega$ with $2<\omega<\omega(\xi)$. Then, there exist infinitely many $k$ for which $\left|Q_{k-1}\right| \leq\left|Q_{k}\right|^{1 / \omega}<\left|Q_{k}\right|^{1 / 2}$. For each such $k$, we choose $j$ such that

$$
\left|S_{j-1}\right|<\left|S_{j}\right| \leq\left|\frac{y_{2} Q_{k}}{x_{2}}\right|^{1 / 2}<\left|S_{j+1}\right| .
$$

The upper right entry $a$ in the unipotent matrix $U(a)$ is chosen to be either the polynomial part $[\rho]$ or $[\rho]+1$. This will depend on the bottom left entry $S_{j} Q_{k}+$ $(-1)^{k-1} Q_{k-1}\left(S_{j} a \pm S_{j-1}\right)$ having absolute value at least $\left|S_{j} Q_{k-1}\right|$ or not for $a=[\rho]$. If

$$
\left|S_{j} Q_{k}+(-1)^{k-1} Q_{k-1}\left(S_{j}[\rho] \pm S_{j-1}\right)\right|<\left|S_{j} Q_{k-1}\right|,
$$

then it cannot be so for $a=[\rho]+1$ as well. It means that the corresponding matrices $\gamma=N_{j} U(a) M_{k}$ will have size at least $\left|S_{j} Q_{k-1}\right|$ for one of those choices. Both $[\rho]$ and $[\rho]+1$ have the same size as $\rho$ equal to $\left|y_{2} Q_{k} / S_{j}\right|$. After this, Lemma 4.7 provides us an $\mathrm{SL}_{2}\left(\mathbb{F}_{q}[T]\right)$ matrix $\gamma$ with height $|\gamma| \leq\left|y_{2} Q_{k}^{3} / x_{2}\right|^{1 / 2}$ and

$$
\begin{aligned}
|\gamma \underline{x}-\underline{y}| & \leq \max \left\{1 /\left|S_{j}\right|, 1\right\}\left|x_{2} y_{2}\right|^{1 / 2}\left|Q_{k}\right|^{-1 / 2} \\
& =\left|x_{2} y_{2}\right|^{1 / 2}\left|Q_{k}\right|^{-1 / 2} \ll_{\underline{x}, \underline{y}}|\gamma|^{-1 / 3} .
\end{aligned}
$$

The lower bound on $\gamma$ established above ensures that we have infinitely many $\mathrm{SL}_{2}\left(\mathbb{F}_{q}[T]\right)$ matrices for which (4.45) holds and $\mu(\underline{x}, \underline{y}) \geq 1 / 3$ here too. 
4.2.3. Uniform exponent. In this subsection, we calculate a lower bound for $\widehat{\mu}(\underline{x}, \underline{y})$ when the target point $\underline{y}$ has an irrational slope $y$. Let $\omega(y)$ be the irrationality measure (with respect to approximation by elements of $K$ ) of $y$.

Lemma 4.9. If $\tau:=\omega(y) /(2 \omega(y)+1), \varepsilon>0$ and $k_{0} \gg_{\varepsilon} 1$ is a natural number, then there exists a matrix $\gamma \in \mathrm{SL}_{2}\left(\mathbb{F}_{q}[T]\right)$ for which

$$
|\gamma| \ll_{\underline{x}, \underline{y}}\left|Q_{k}\right|^{2} \text { and }|\gamma \underline{x}-\underline{y}| \leq\left|Q_{k}\right|^{\tau-1+\varepsilon} .
$$

Proof. Observe that $\tau$ is in the range $[1 / 3,1 / 2]$ as $\omega(y) \in[1, \infty]$. Much like [10], our choice of the indices $j$ and $k$ in the construction of $\gamma$ is governed by

$$
\left|S_{j}\right| \leq\left|Q_{k}\right|^{\tau}<\left|S_{j+1}\right|
$$

so that both of them go to infinity together. For $\omega<\infty$, we let $\omega>\omega(y)$ but sufficiently close to make sure that $1 / \omega>1 / \omega(y)-\varepsilon / \tau$. Then, $\left|S_{j}\right| \geq\left|S_{j+1}\right|^{1 / \omega}$ for all $j \gg 1$ from $\oint 2$ implying that one has $\left|S_{j}\right| \geq\left|Q_{k}\right|^{\tau / \omega(y)-\varepsilon}$ for all large $k$. Now, notice that this lower bound is trivially true when $\omega(y)=\infty$.

With $j$ and $k$ related by (4.46), Lemma 4.7 gives us an $\mathrm{SL}_{2}\left(\mathbb{F}_{q}[T]\right)$ matrix $\gamma$ such that

$$
\begin{gathered}
|\gamma| \ll_{\underline{x}, \underline{y}} \max \left\{\left|S_{j} Q_{k}\right|,\left|Q_{k} Q_{k-1}\right|\right\} \leq\left|Q_{k}\right|^{2} \text {, and } \\
|\gamma \underline{x}-\underline{y}| \ll_{\underline{x}, \underline{y}} \max \left\{1 /\left|S_{j} S_{j+1}\right|,\left|S_{j} / Q_{k}\right|\right\} \leq\left|Q_{k}\right|^{\tau-1+\varepsilon} .
\end{gathered}
$$

The last dependence on $\underline{x}$ and $\underline{y}$ is absorbed in the rising $\varepsilon$-powers of $\left|Q_{k}\right|$ 's.

Given any $H \gg 1$, we pick the integer $k$ for which $c_{0}\left|Q_{k}\right|^{2} \leq H<c_{0}\left|Q_{k+1}\right|^{2}$ where $c_{0}$ is the implied constant in the upper bound on $|\gamma|$ obtained in Lemma 4.9. When $H$ and consequently $k$ is large enough in terms of $\varepsilon>0$, it follows from the definition of $\omega(\xi)$ that $\left|Q_{k+1}\right|^{2} \leq\left|Q_{k}\right|^{2 \omega(\xi)+\varepsilon}$. Hence, we get a matrix $\gamma$ with $|\gamma| \leq H$ and also,

$$
|\gamma \underline{x}-\underline{y}| \leq\left|Q_{k}\right|^{-(1-\tau-\varepsilon)} \ll H^{-(1-\tau-\varepsilon) /(2 \omega(\xi)+\varepsilon)}
$$

assuming $\varepsilon<1 / 2$. In other words, we have

$$
\widehat{\mu}(\underline{x}, \underline{y}) \geq \frac{1-\tau-\varepsilon}{2 \omega(\xi)+\varepsilon}
$$

where $\varepsilon$ may be erased from the numerator and denominator by taking the limit as $\varepsilon$ tends to 0 . This gives us the second part of Proposition 4.8 .

4.2.4. A generic upper bound. We now focus on having an upper bound for the asymptotic exponent $\mu(\underline{x}, \underline{y})$. This shall be possible for us only for a co-null set consisting of target vectors whose slope has irrationality measure 1 . The lemma below rewrites a matrix $\gamma$ which helps $\underline{x}$ to reach close to $\underline{y}$ as a product of the convergent matrices $N_{j}, M_{k}$ and some $G \in \mathrm{SL}_{2}\left(\mathbb{F}_{q}[T]\right)$ for some $j$ and $k$ so that we have good control over the entries of $G$.

Lemma 4.10. If $\mu \in[0,1]$ and $H \gg_{\underline{x}} 1$ are real numbers such that $\gamma$ satisfies both $|\gamma| \leq q H$ and $|\gamma \underline{x}-\underline{y}| \leq H^{-\mu}, \underline{k}$ is the index for which $\left|Q_{k-1} Q_{k}\right| \leq H \leq$ $\left|Q_{k} Q_{k+1}\right|$ and $j$ is large enough to guarantee $\left|S_{j}\right| \geq H^{\mu / 2}$, then $\gamma=N_{j} G M_{k}$ for some $\mathrm{SL}_{2}\left(\mathbb{F}_{q}[T]\right)$ matrix $G$ whose first and second columns are respectively bounded above in size by

$$
c_{1}\left|S_{j} / Q_{k}\right| H^{1-\mu} \text { and } c_{1}\left|S_{j} Q_{k}\right| H^{-\mu},
$$


INHOMOGENEOUS DIOPHANTINE APPROXIMATION OVER POWER SERIES FIELDS 19

where $c_{1}:=\max \left\{q, 1 /\left|x_{2}\right|,\left|y_{2} / x_{2}\right|, q /\left|y_{2}\right|\right\}$.

Proof. The matrix $\gamma$ is again taken to be

$$
\left(\begin{array}{ll}
V_{1} & U_{1} \\
V_{2} & U_{2}
\end{array}\right)
$$

and then, the components $\Lambda_{i}$ of the difference $\gamma \underline{x}-\underline{y}$ are given by (4.11). Our hypothesis implies that $\max \left\{\left|\Lambda_{1}\right|,\left|\Lambda_{2}\right|\right\} \leq H^{-\mu}$. From the argument involved in (4.25), one has $\left|V_{1} y_{2}-V_{2} y_{1}\right| \leq q H^{1-\mu}$ and similarly,

$$
\left|U_{1} y_{2}-U_{2} y_{1}\right| \leq \max \left\{|\xi|, H^{1-\mu}\right\}=q H^{1-\mu}
$$

as we earlier took $|\xi| \leq 1$. The convergent matrix $N_{j}$ is factored in first so that

$$
\begin{aligned}
\widetilde{\gamma} & =\left(\begin{array}{cc}
\widetilde{V}_{1} & \widetilde{U}_{1} \\
\widetilde{V}_{2} & \widetilde{U}_{2}
\end{array}\right):=N_{j}^{-1} \gamma=\left(\begin{array}{cc}
\widetilde{S}_{j-1} V_{1}-\widetilde{R}_{j-1} V_{2} & \widetilde{S}_{j-1} U_{1}-\widetilde{R}_{j-1} U_{2} \\
-S_{j} V_{1}+R_{j} V_{2} & -S_{j} U_{1}+R_{j} U_{2}
\end{array}\right) \\
& =\frac{1}{y_{2}}\left(\begin{array}{cc}
\widetilde{S}_{j-1}\left(V_{1} y_{2}-V_{2} y_{1}\right)+V_{2}\left(\widetilde{S}_{j-1} y_{1}-\widetilde{R}_{j-1} y_{2}\right) & \widetilde{S}_{j-1}\left(U_{1} y_{2}-U_{2} y_{1}\right)+U_{2}\left(\widetilde{S}_{j-1} y_{1}-\widetilde{R}_{j-1} y_{2}\right) \\
-S_{j}\left(V_{1} y_{2}-V_{2} y_{1}\right)-V_{2}\left(S_{j} y_{1}-R_{j} y_{2}\right) & -S_{j}\left(U_{1} y_{2}-U_{2} y_{1}\right)-U_{2}\left(S_{j} y_{1}-R_{j} y_{2}\right)
\end{array}\right) .
\end{aligned}
$$

Since $\left|S_{j} y-R_{j}\right|<\left|\widetilde{S}_{j-1} y-\widetilde{R}_{j-1}\right|=1 /\left|S_{j}\right|$, we get

$$
|\widetilde{\gamma}| \leq q \cdot \max \left\{\left|S_{j} / y_{2}\right| H^{1-\mu}, H /\left|S_{j}\right|\right\}=q \cdot \max \left\{\left|y_{2}\right|^{-1}, 1\right\}\left|S_{j}\right| H^{1-\mu}
$$

for all $\mathrm{j}$ such that $\left|S_{j}\right| \geq H^{\mu / 2}$. One also has

$$
\begin{aligned}
\widetilde{\gamma} \underline{x} & =x_{2}\left[\begin{array}{c}
\widetilde{V}_{1} \xi+\widetilde{U}_{1} \\
\widetilde{V}_{2} \xi+\widetilde{U}_{2}
\end{array}\right]=N_{j}^{-1}\left(\underline{y}+\left[\begin{array}{c}
\Lambda_{1} \\
\Lambda_{2}
\end{array}\right]\right) \\
& =\left[\begin{array}{c}
y_{2}\left(\widetilde{S}_{j-1} y-\widetilde{R}_{j-1}\right)+\widetilde{S}_{j-1} \Lambda_{1}-\widetilde{R}_{j-1} \Lambda_{2} \\
y_{2}\left(-y S_{j}+R_{j}\right)-\left(S_{j} \Lambda_{1}-R_{j} \Lambda_{2}\right)
\end{array}\right]
\end{aligned}
$$

which implies that $|\widetilde{\gamma} \underline{x}| \leq \max \left\{\left|y_{2} / S_{j}\right|,\left|S_{j}\right| H^{-\mu}\right\} \leq \max \left\{\left|y_{2}\right|, 1\right\}\left|S_{j}\right| H^{-\mu}$. Let us further append $M_{k}^{-1}$ to the right of $\widetilde{\gamma}$ and denote

$$
\begin{aligned}
G & :=N_{j}^{-1} \gamma M_{k}^{-1}=\left(\begin{array}{ll}
\widetilde{V}_{1} & \widetilde{U}_{1} \\
\widetilde{V}_{2} & \widetilde{U}_{2}
\end{array}\right)\left(\begin{array}{cc}
(-1)^{k} P_{k-1} & P_{k} \\
(-1)^{k} Q_{k-1} & Q_{k}
\end{array}\right) \\
& =\left(\begin{array}{ll}
(-1)^{k}\left(P_{k-1} \widetilde{V}_{1}+Q_{k-1} \widetilde{U}_{1}\right) & P_{k} \widetilde{V}_{1}+Q_{k} \widetilde{U}_{1} \\
(-1)^{k}\left(P_{k-1} \widetilde{V}_{2}+Q_{k-1} \widetilde{U}_{2}\right) & P_{k} \widetilde{V}_{2}+Q_{k} \widetilde{U}_{2}
\end{array}\right) .
\end{aligned}
$$

The top and bottom entries of the left column of this matrix are the same as

$$
-\widetilde{V}_{i}\left(Q_{k-1} \xi-P_{k-1}\right)+Q_{k-1}\left(\widetilde{V}_{i} \xi+\widetilde{U}_{i}\right)
$$

for $i$ equal to 1 and 2, respectively and upto multiplication by \pm 1 . Both of these expressions are bounded above by

$$
\max \left\{\frac{|\widetilde{\gamma}|}{\left|Q_{k}\right|}, \frac{\left|Q_{k-1}\right| \widetilde{\gamma} \underline{x}||}{\left|x_{2}\right|}\right\} \leq \max \left\{q, \frac{1}{\left|x_{2}\right|},\left|\frac{y_{2}}{x_{2}}\right|, \frac{q}{\left|y_{2}\right|}\right\}\left|S_{j} / Q_{k}\right| H^{1-\mu} .
$$

Insofar as the other column is concerned,

$$
\begin{aligned}
\left|P_{k} \widetilde{V}_{i}+Q_{k} \widetilde{U}_{i}\right| & =\left|-\widetilde{V}_{i}\left(Q_{k} \xi-P_{k}\right)+Q_{k}\left(\widetilde{V}_{i} \xi+\widetilde{U}_{i}\right)\right| \\
& \leq \max \left\{|\widetilde{\gamma}| /\left|Q_{k+1}\right|,\left|Q_{k}\right||\widetilde{\gamma} \underline{x}| /\left|x_{2}\right|\right\} \\
& \leq \max \left\{q, 1 /\left|x_{2}\right|,\left|y_{2} / x_{2}\right|, q /\left|y_{2}\right|\right\}\left|S_{j} Q_{k}\right| H^{-\mu}
\end{aligned}
$$


employing the set of inequalities $\left|Q_{k-1} Q_{k}\right| \leq H \leq\left|Q_{k} Q_{k+1}\right|$ to the fullest.

Next, we fix $\underline{x} \in K_{\infty}^{2} \backslash\{\underline{0}\}, y \in K_{\infty} \backslash K$ such that $\omega(y)=1$ and $\mu>1 / 2$. Consider any compact subset $\mathcal{C} \subset K_{\infty}{ }^{t}(y, 1) \backslash\{\underline{0}\}$. Following Laurent and Nogueira [10], we show that the set

$$
\mathcal{C}_{\mu}:=\left\{\underline{y} \in \mathcal{C}|| \gamma \underline{x}-\left.\underline{y}|\leq| \gamma\right|^{-\mu} \text { for infinitely many } \gamma \in \mathrm{SL}_{2}\left(\mathbb{F}_{q}[T]\right)\right\}
$$

has one-dimensional Lebesgue measure 0 . Since $\mathcal{C}$ and $\mu$ were chosen arbitrarily, one has that for almost all points $\underline{y} \in K_{\infty}{ }^{t}(y, 1)$, the asymptotic Diophantine exponent

$$
\mu(\underline{x}, \underline{y}) \leq 1 / 2 .
$$

Suppose $\underline{y} \in \mathcal{C}_{\mu}$ and $|\gamma| \gg 1$. Our integers $k \geq 1$ and $n \geq 0$ are such that

$$
\begin{aligned}
\left|Q_{k-1} Q_{k}\right| & <|\gamma| \leq\left|Q_{k} Q_{k+1}\right| \text { and } \\
q^{n}\left|Q_{k-1} Q_{k}\right| & <|\gamma| \leq q^{n+1}\left|Q_{k-1} Q_{k}\right| .
\end{aligned}
$$

If $H$ is taken to be $q^{n}\left|Q_{k-1} Q_{k}\right|$, we get $|\gamma| \leq q H$ and $|\gamma \underline{x}-\underline{y}| \leq|\gamma|^{-\mu}<$ $H^{-\mu}$. Now, let $j$ be the smallest number for which $\left|S_{j}\right| \geq H^{\mu / 2}$. As $\omega(y)=1$, this denominator $S_{j}$ cannot be too large in size compared to $S_{j-1}$. Namely, $\left|S_{j}\right| \leq\left|S_{j-1}\right|^{1+2 \varepsilon / \mu}<H^{\mu / 2+\varepsilon}$ for any $\varepsilon>0$ and all but finitely many $j$ 's depending on $\varepsilon$. This dependence has already been accounted for while choosing $|\gamma|$ sufficiently big. Subsequently, Lemma 4.10 informs us of the existence of a matrix decomposition $\gamma=N_{j} G M_{k}$ with the first column of $G$ bounded by $c_{2}\left|S_{j} / Q_{k}\right| H^{1-\mu} \leq c_{2} H^{1-\mu / 2+\varepsilon} /\left|Q_{k}\right|$ and the second column by $c_{2}\left|Q_{k}\right| H^{-\mu / 2+\varepsilon}$. The new constant $c_{2}=\max _{\underline{y} \in \mathcal{C}} \max \left\{q, 1 /\left|x_{2}\right|,|\underline{y}| /\left|x_{2}\right|, q /|\underline{y}|\right\}$ is a function of $\mathcal{C}$ alone for a fixed $\underline{x}$.

Proposition 4.11. The number of $\mathrm{SL}_{2}\left(\mathbb{F}_{q}[T]\right)$ matrices with first and second columns (rows) respectively bounded by $B_{1}$ and $B_{2}$ is at most $\mathcal{O}\left(B_{1} B_{2}\right)$.

Proof. Without loss of generality, we may take $B_{1} \leq B_{2}$ or else interchange their roles. Let us assume that the norm of the first column is realized by the entry $f$ in the top row and equals $q^{i}$ for some $i$ such that $q^{i} \leq B_{1}$. Given any such polynomial $f$, the number of allowed entries in the second row of the first column is at most $q \Phi(f)$ where $\Phi(f)$ denotes the number of elements in the multiplicative group $\left(\mathbb{F}_{q}[T] / f \mathbb{F}_{q}[T]\right)^{*}$. With $a_{1,1}$ and $a_{2,1}$ fixed, there is a unique solution to the equation $a_{1,2} a_{2,1} \equiv-1$ in $\left(\mathbb{F}_{q}[T] / a_{1,1} \mathbb{F}_{q}[T]\right)^{*}$ corresponding to which we have a unique $a_{2,2}$ satisfying the determinant one condition. Hence, the number of polynomial vectors ${ }^{t}\left(a_{1,2}, a_{2,2}\right)$ such that $a_{1,1} a_{2,2}-a_{1,2} a_{2,1}=1$ and $\max \left\{\left|a_{1,2}\right|,\left|a_{2,2}\right|\right\} \leq B_{2}$ is at most $q B_{2} /|f|$. Combining all the choices made, we have our number as

$$
\ll \sum_{\substack{i \geq 0, q^{i} \leq B_{1}}} \sum_{\operatorname{deg} f=i} q \Phi(f) \frac{q B_{2}}{|f|} \ll_{q} B_{2} \sum_{\substack{i \geq 0, q^{i} \leq B_{1}}} \frac{1}{q^{i}} \sum_{\operatorname{deg} f=i} \Phi(f) .
$$

The value of the last inner sum can be obtained from [14, Proposition 2.7] to be $q^{2 i}(q-1)$. Thereafter, the claim is easily seen to be true.

For the matrices $G$ which arose in Lemma 4.10, there are $\mathcal{O}\left(c_{2}^{2} H^{1-\mu+2 \varepsilon}\right)$ possibilities with 0 being the obvious upper bound if either of $c_{2} H^{1-\mu / 2+\varepsilon} /\left|Q_{k}\right|$ or $c_{2}\left|Q_{k}\right| H^{-\mu / 2+\varepsilon}$ is less than 1 . The ball of diameter $\leq H^{-\mu}$ centered around the point $\gamma \underline{x} \in K_{\infty}^{2}$ will not intersect the line $K_{\infty}{ }^{t}(y, 1)$ in a ball of diameter any bigger 
than $H^{-\mu}$. If we fix $k$ and $n$ in (4.60), we have not more than $\mathcal{O}_{\mathcal{C}, \underline{x}}\left(H^{1-\mu+2 \varepsilon}\right)$ such matrices $\gamma \in \mathrm{SL}_{2}\left(\mathbb{F}_{q}[T]\right)$. Otherwise said, our target point $\underline{y}$ belongs to some union of balls in $K_{\infty}{ }^{t}(y, 1)$ whose one-dimensional Lebesgue measure is

$$
\ll_{\mathcal{C}, \underline{x}} H^{1-2 \mu+2 \varepsilon}=\left(q^{n}\left|Q_{k-1} Q_{k}\right|\right)^{1-2 \mu+2 \varepsilon}
$$

by the definition of $H$. When $\varepsilon$ is small enough so that $1-2 \mu+2 \varepsilon<0$ (possible since $\mu>1 / 2$ ), the term in (4.62) sums up to something finite when we consider all $k \geq 1$ and $n \geq 0$. The Borel-Cantelli lemma tells us that the measure of the set $\mathcal{C}_{\mu} \subset K_{\infty}{ }^{t}(y, 1)$ is zero.

\section{REFERENCES}

[1] Y. Bugeaud and M. Laurent. On exponents of homogeneous and inhomogeneous Diophantine approximation. Mosc. Math. J., 5(4):747-766, 2005.

[2] Y. Bugeaud and Z. L. Zhang. On homogeneous and inhomogeneous Diophantine approximation over the fields of formal power series. (To appear in Pacific J. Math.).

[3] J. W. S. Cassels. An introduction to Diophantine approximation. Cambridge Tracts in Mathematics and Mathematical Physics, No. 45. Cambridge University Press, New York, 1957.

[4] M. Fuchs. Metrical theorems for inhomogeneous Diophantine approximation in positive characteristic. Acta Arith., 141(2): 191-208, 2010.

[5] A. Ghosh, A. Gorodnik and A. Nevo. Best possible rates of distribution of dense lattice orbits in homogeneous spaces. (To appear in J. Reine Angew. Math.).

[6] A. Ghosh, A. Gorodnik and A. Nevo. Diophantine approximation exponents on homogeneous varieties. In Recent trends in ergodic theory and dynamical systems, Contemp. Math. 631: 181-200, 2015

[7] A. Ya. Khinchin. Continued fractions. The University of Chicago Press, Chicago, Ill.-London, 1964.

[8] D. H. Kim and H. Nakada. Metric inhomogeneous Diophantine approximation on the field of formal Laurent series. Acta Arith., 150(2): 129-142, 2011.

[9] S. Kristensen. Metric inhomogeneous Diophantine approximation in positive characteristic. Math. Scand., 108(1): 55-76, 2011.

[10] M. Laurent and A. Nogueira. Approximation to points in the plane by $\operatorname{SL}(2, \mathbb{Z})$ orbits. J. London Math. Soc., 85(2): 409-429, 2012.

[11] C. Ma and W. Y. Su. Inhomogeneous Diophantine approximation over the field of formal Laurent series. Finite Fields Appl., 14(2): 361-378, 2008.

[12] F. Maucourant and B. Weiss. Lattice actions on the plane revisited. Geom. Dedicata, 157: 1-21, 2012.

[13] M. Pollicott. Rates of convergence for linear actions of cocompact lattices on the complex plane. Integers, 11B: Paper No. A12, 2011.

[14] M. Rosen. Number theory in function fields, Springer-Verlag, New York, 2002.

[15] W. M. Schmidt. On continued fractions and diophantine approximation in power series fields. Acta Arith., 95(2): 139-166, 2000.

[16] L. Singhal. Diophantine exponents for standard linear actions of $\mathrm{SL}_{2}$ over discrete rings in $\mathbb{C}$. Acta Arith., 177(1): 53-73, 2017.

[17] V. G. Sprindzhuk. Mahler's problem in metric number theory. American Mathematical Soc., 1969. 
[18] Z. Y. Zheng. Simultaneous Diophantine Approximation in Function Fields. arXiv preprint arXiv:1711.03721, 2017.

Université de Strasbourg, 7, rue René Descartes, 67084 Strasbourg, France

E-mail address: bugeaud@math.unistra.fr

Beijing International Center for Mathematical Research, Peking University, 100871 BEIJing, P. R. CHINA

E-mail address: 1sd@bicmr.pku.edu.cn

School of Mathematical Sciences, Henan Institute of Science and Technology, 453003 Xinxiang, P. R. CHINA

E-mail address: zhliang_zhang@hotmail.com 